\title{
Growth of a Richtmyer-Meshkov Turbulent Layer After Reshock ${ }^{\dagger}$
}

\author{
B.Thornber* and D.Drikakis \\ Dept. of Fluid Mechanics 85 Computational Science, \\ Cranfield University, \\ Cranfield, MK43 OAL, UK \\ D.L. Youngs and R.J.R. Williams \\ AWE, Aldermaston, Reading RG7 $4 P R, U K$ \\ ${ }^{\dagger}$ Contains material (C)British Crown Copyright 2011/MoD
}

(Dated: July 23, 2011)

\begin{abstract}
This paper presents a numerical study of a reshocked turbulent mixing layer using high-order accurate Implicit Large-Eddy-Simulations (ILES). Existing theoretical approaches are discussed, and the theory of Youngs [1] is extended to predict the behaviour of a reshocked mixing layer formed initially from a shock interacting with a broadband instability. The theory of Mikaelian [2] is also extended to account for molecular mixing in the single-shocked layer prior to reshock. Simulations are conducted for broadband and narrowband initial perturbations and results for the growth rate of the reshocked layer and the decay rate of turbulent kinetic energy show excellent agreement with the extended theoretical approach. Reshock causes a marginal decrease in mixing parameters for the narrowband layer, but a significant increase for the broadband initial perturbation. The layer properties are observed to be very similar post-reshock, however, the growth rate exponent for the mixing layer width is higher in the broadband case, indicating that the reshocked layer still has a dependence (although weakened) on the initial conditions. These results have important implications for Unsteady Reynolds Averaged Navier Stokes modelling of such instabilities.
\end{abstract}

PACS numbers: 47.27.wj,47.27.ep,47.27.Jv,47.40.Nm,47.20.Ma

*Electronic address: b.j.r.thornber@cranfield.ac.uk 


\section{INTRODUCTION}

The Richtmyer-Meshkov (RM) instability occurs when an interface between two fluid layers of different thermodynamic properties undergoes impulsive acceleration, typically caused by an incident shock wave $[3,4]$. This instability is important in the prediction of the evolution of supernovae $[5,6]$, in the wakes of jet engines, mixing in scramjet combustion chambers, and in predicting Inertial Confinement Fusion (ICF) capsule performance [7].

Once the instability has been triggered, it will grow at first linearly, and then transition to a non-linear state characterised by mushroom shaped bubbles (light fluid moving into heavier fluid) and spikes (heavy fluid moving into light fluid). At this point the KelvinHelmholtz instability acts on the shear layers along the interface, causing a rapid transition to turbulence. Once a fully turbulent layer is established, the statistics of the mixing layer tend towards a self similar state [8].

Under reshock, this turbulent layer caused by the first shock wave then undergoes a second impulsive acceleration. This acceleration can be caused by reflection of the incident shock wave from the end wall of a shock tube, or by an outwardly propagating shock wave in the case of a cylindrical/spherical implosion. Experimental observations show that the second shock wave significantly increases the layer growth rate [9-13]. There is general consensus that the growth of the mixing layer is described by $W \propto t^{\theta}$. Analytical results suggest either linear growth in time [2], a growth rate of $\theta=2 / 3-\mu[14,15]$ where $\mu$ is a correction dependent on the level of turbulent dissipation, $\theta=0.4$ [16] or even logarithmic [16-18].

Experimental results are difficult to measure due to the complex flow physics such as fluid compressibility (including shocks), multiple gases, uncertainty in the form of the initial perturbation, wall effects, and separating the growth of the mixing layer due to expansion waves from turbulent growth. Results by Vetter \& Sturtevant [11] and Leinov et al. [13] indicated approximate linear growth after reshock $(\theta=1)$. Leinov et al also investigated the influence of the mixing layer width pre-reshock on the resultant growth rate showing that it had negligible effect, whereas the growth rate was linearly proportional to the velocity jump at the interface. However, experiments by Houas and Chemouni [19] showed better agreement to a power law behaviour $\theta=2 / 3$ rather than the linear solution, and Prasad et al. [20] suggest growth of $\theta=0.26-0.33$. Recently, the gas curtain experiments of 
Balakumar et al. [12] also show clear nonlinear behaviour of the reshocked layer, where the initial perturbation consists of a single dominant wavelength.

Several numerical studies have examined cases of a reshock of an RM-induced turbulent mixing layer, principally focusing on the various shock tube experiments. Large Eddy Simulations (LES) by Hill et al. [21] matched the post-reshock growth measured in the experiments of Vetter \& Sturtevant to within $\pm 10 \%$. Further LES by Latini et al. [22] also match the experimental measurements, however again only for the reshocked layer - prior to this the growth rate is highly dependent on the initial conditions [22]. Schilling et al. [23] investigated a two dimensional reshocked single mode case and found that of the models which do not depend on the wave number, $k$, (hence could be easily applied to a multimode case) the Mikaelian model [2] captured the early time reshock behaviour the most accurately.

This paper examines, using high-order accurate ILES methods, the growth rate of a reshocked mixing layer based on initially narrowband and broadband perturbations. This study builds upon the previous study of Thornber et al. [8] which focused on the selfsimilar behaviour of a single-shock RM instability. The broadband simulation employs a perturbation spectrum typical of the finish of an ICF capsule, where $P(k) \propto k^{-2}$ [24]. The aim is to study the growth rate in isolation of other fluid dynamic phenomena experienced within the shock-tube experiments, for example reflected rarefaction waves. Hence, the two shocks are generated numerically within the computational domain with non-reflective boundary conditions to allow the reflected shock/expansions to exit the domain unimpeded.

Section II details the governing equations, numerical methods and initial/boundary conditions for the reshock test case. Section III discusses current theoretical approaches, and proposes an extension of the theory of Youngs [1] for broadband perturbations with reshock. The results are presented in Section IV, and conclusions summarised in the final section.

\section{GOVERNING EQUATIONS AND NUMERICAL METHODS}

\section{A. Governing Equations}

The computations presented here employ the multi-component Euler equations, where viscosity is assumed negligible $(R e \rightarrow \infty)$. This involves solving in each principal direction the following governing equations, 


$$
\frac{\partial \mathbf{U}}{\partial t}+\frac{\partial \mathbf{F}}{\partial x}=0
$$

where,

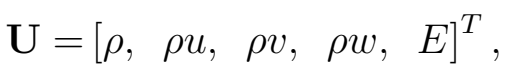

$$
\begin{aligned}
& \mathbf{F}=\left[\rho u, \quad \rho u^{2}+p, \quad \rho u v, \quad \rho u w, \quad(E+p) u\right]^{T}, \\
& E=\rho e+0.5 \rho\left(u^{2}+v^{2}+w^{2}\right),
\end{aligned}
$$

and $\rho, E, e, u, v$, and $w$ are the density, total energy, specific internal energy per unit volume, and the Cartesian velocity components, respectively. Throughout this paper, it is assumed that the fluid satisfies the ideal gas equation of state $p=\rho e(\gamma-1)$, where $\gamma=5 / 3$ is the ratio of specific heats and is identical for both fluids.

For the multiple component simulations presented here, the model proposed by Allaire et al. [25] is employed in lieu of the continuity equation, replacing it with the following equation set (for the sake of simplicity the model is presented in 1D) for two species,

$$
\begin{aligned}
\frac{\partial \alpha_{1} \rho_{1}}{\partial t}+\frac{\partial \alpha_{1} \rho_{1} u}{\partial x} & =0 \\
\frac{\partial \alpha_{2} \rho_{2}}{\partial t}+\frac{\partial \alpha_{2} \rho_{2} u}{\partial x} & =0 \\
\frac{\partial \alpha_{1}}{\partial t}+u \frac{\partial \alpha_{1}}{\partial x} & =0
\end{aligned}
$$

where $\alpha_{1,2}$ are the volume fractions of the light and heavy fluids. This choice of governing equations proves superior in numerical test cases to mass fraction based approaches and other volume fraction methods for sharp advection of multiple species with different thermodynamic properties [26]. Note that it is assumed that if two fluids are present in one cell the mixture has one characteristic velocity, and both species are in pressure equilibrium.

\section{B. Numerical Approach}

The numerical approach employed is based on a finite volume Godunov-type method, henceforth labelled as CNS3D [27-29]. This employs fifth order spatial accuracy through a one dimensional implementation of the limiter proposed by Kim and Kim [30], with the 
second order accurate extended stability Runge-Kutta scheme of Spiteri and Ruuth [31]. The governing equations for a multicomponent flow are solved using the approach proposed in Allaire et al. [25], however, the current work employs the HLLC approximate Riemann solver [32].

To improve the performance of the baseline fifth order method, the modified reconstruction method of [29] is employed. This modification is based on analysis presented in [33] which shows that standard Godunov methods dissipation turbulent kinetic energy excessively at low Mach due to the artificially high amplitude of the velocity jumps at the cell interfaces prior to solving the Riemann problem. This is supported strongly by analysis of simulations of homogeneous decaying turbulence [34, 35]. The modified reconstruction method effectively progresses to central differencing of the reconstructed left and right quantities as the Mach number tends towards zero, giving uniform dissipation of turbulent kinetic energy. This modification is also supported in a recent analysis by Dellacherie et al. [36, 37].

The simulations use the Implicit LES approach [28, 38, 39], where the numerical methods is designed in such a manner to provide realistic dissipation of turbulent kinetic energy. An important consequence of the modification to the spatial reconstruction method is that the dissipation rate is now proportional to the cube of the velocity divided by the cell size, as required for an implicit sub-grid scale model based on Kolmogorov's analysis of dissipation in homogeneous turbulence [40]. As it is very unlikely that this model perfectly represents the subgrid scales, the simulations employ high resolution grids in order to provide sufficient scale separation between the largest and smallest flow features. When employed in a free shear flow, this wide range of scale separation (large grid sizes) permits the large features to evolve independently of numerical dissipation.

This exact numerical method has been validated in one-, two- and three dimensional test cases [29, 39, 41]; low-speed internal separated flows [42]; cavity aeroacoustics [43, 44]; ship airwakes [45]; hypersonic flows, including ablation [46]; and turbulent mixing induced by RM instability $[8,47]$.

\section{Initial and Boundary Conditions}

Two different initial conditions are employed for the reshock study, using the data produced in a previous study on the RM instability [8]. As this is a study of reshock, the initial 
profiles of density, volume fractions, velocity and pressure from the single shock study are used for the reshock case. The single shock study examined the onset of self-similarity of the mixing layer through examination of several mixing parameters, the variation of the mixing layer growth exponent $\theta$ and the decay exponent of turbulent kinetic energy. The initial conditions for this study are chosen at the onset of self-similarity after the passage of the first shock, where all parameters are now self-similar, but before the mixing layer width has grown to a significant fraction of the domain size. The onset of self-similarity is identified as the time when the mixing parameters $\Theta$ and $\Xi$ (see Section IV C for their definition) are within $\pm 5 \%$ of their expected self similar values identified in the previous single shock study, and that the decay of turbulent kinetic energy has reached a self-similar power law form [8]. This gives the correct variation of density and volume fraction across the mixing layer which governs the magnitude of the vorticity deposited in the mixing layer due to reshock.

The passage of the first shock is represented by an equivalent velocity perturbation derived from an assumed surface perturbation power spectrum [8]. Considering a single mode from a multimode perturbation of the form

$$
\begin{array}{r}
A(y, z)=\sum_{m, n=0}^{N} a_{m, n} \cos \left(k_{0} m y\right) \cos \left(k_{0} n z\right)+b_{m, n} \cos \left(k_{0} m y\right) \sin \left(k_{0} n z\right)+ \\
c_{m, n} \sin \left(k_{0} m y\right) \cos \left(k_{0} n z\right)+d_{m, n} \sin \left(k_{0} m y\right) \sin \left(k_{0} n z\right)
\end{array}
$$

which considers modes with wave number $m$ in the $y$ direction and $n$ in the $z$ direction where $x$ is in the direction of shock propagation, $y$ and $z$ range from 0 to $L$. Following the initial conditions employed in [8], to ensure an initially divergence-free velocity field a vector potential $\mathbf{A}$ is defined such that $\mathbf{u}=\nabla \times \mathbf{A}$ where $\mathbf{A}$ consists of two non-zero components $\left(A_{y}\right.$ and $\left.A_{z}\right)$ given by

$$
\begin{array}{r}
A_{y}(x, y, z)=\phi_{0} \exp \left(-k\left|x-x_{1}\right|\right) \frac{k_{z}}{k}\left[b \cos \left(k_{y} y\right) \cos \left(k_{z} z\right)-a \cos \left(k_{y} y\right) \sin \left(k_{z} z\right)+\right. \\
\left.d \sin \left(k_{y} y\right) \cos \left(k_{z} z\right)-c \sin \left(k_{y} y\right) \sin \left(k_{z} z\right)\right] . \\
A_{z}(x, y, z)=\phi_{0} \exp \left(-k\left|x-x_{1}\right|\right) \frac{k_{y}}{k}\left[-c \cos \left(k_{y} y\right) \cos \left(k_{z} z\right)-d \cos \left(k_{y} y\right) \sin \left(k_{z} z\right)+\right. \\
\left.a \sin \left(k_{y} y\right) \cos \left(k_{z} z\right)+b \sin \left(k_{y} y\right) \sin \left(k_{z} z\right)\right] .
\end{array}
$$


where the initial location of the mixing layer is at $x=x_{1}$, where $\phi_{0}$ is a parameter which controls the magnitude of the perturbation. For RM, the magnitude of the initial perturbation is determined from linear theory, giving $\phi_{0}=A t^{+} \Delta u$. The velocity perturbations decay exponentially away from the interface in agreement with incompressible theory (an approximation to the compressible theory where the decay is slower [48]). Finally, an additional exponential decay is added to ensure that the velocities become zero at the boundaries of the domain. The coefficients $a-d$ are defined as $a_{m, n}=\mathcal{R} S(m) S(n) \sigma_{m, n}$, where $S(p)=1 / \sqrt{2}$ if $p=0,1$ if $p>0, \mathcal{R}$ is a random number picked from a Gaussian distribution, and $\sigma_{m, n}$ is the standard deviation of a mode with wave number $m$ in the $y$ direction and $n$ in the $z$ direction.

Two different initial perturbation power spectra are considered, the first is a narrowband spectrum where $P(k) \propto C$ for modes of wavelength $16 \Delta x \leq \lambda \leq 32 \Delta x$ where $\Delta x$ is the grid spacing which will be referred to as the 'narrowband mixing layer' throughout the paper. The second is a more inhomogeneous turbulent layer generated by a broadband initial perturbation with a power spectrum of the form $P(k) \propto C k^{-2}$ for modes of wavelength $8 \Delta x \leq \lambda \leq \pi$. This perturbation was chosen as it is typical of the surface finish measured for inertial confinement fusion capsules [24], and will be referred to as a 'broadband mixing layer'. The minimum wavelengths have been chosen based on previous experience demonstrating the minimum requirements for correct resolution of the growth of the resulting turbulent mixing layer [8].

The coefficients $C$ are scaled in the narrowband initial condition such that $\sigma^{2}=$ $\int_{k_{\min }}^{k_{\max }} P(k) d k=0.1 \lambda_{\min }$. As the initial modes present in the problem are in a relatively narrow range then this is sufficient to ensure linearity of the initial wavelengths.

For the broadband case the shorter wavelengths are 'more non-linear' than the longer wavelengths hence care must be taken to ensure linearity at the shortest wavelengths. The value of $C$ in the broadband case is determined by specifying that the in-band amplitude $a_{k}$ of the highest wavenumber remain remains linear. Due to the interference of nearby modes, $a_{k}$ is defined as an integral of individual mode amplitudes over a band of width $k$ surrounding mode $k$ as detailed by Haan [49] and utilised previously in [8]. In the simulations presented here, $k a_{k}=1 / 2$ for the highest wavenumber resolved on the grid.

The simulations for the evolution of the mixing layer after the first shock (i.e. the evolution of the velocity perturbation) were run on grids of $512^{3}$ for the narrowband mixing 
layer and $720 \times 2048 \times 2048$ for the broadband layer. This ensured that the broadband layer had a sufficiently wide spectral range to achieve self-similarity, and that the narrowband layer was well resolved [8].

Once a self-similar regime has been achieve for each mixing layer from the initial velocity perturbation, the simulation is stopped. Figure 1 shows the initial conditions for the reshock simulation. The turbulent mixing layer from the first simulation is used to initialise the second simulation. In both cases the initial density ratio is $3: 1$, and the reshock is from the heavy to the light gas. The light gas and unshocked heavy gas are given an initial velocity into the shock such that the reshocked mixing layer remains stationary in the chosen frame of reference. This is illustrated schematically in Figure 1. The shock strength is Mach 1.85, equivalent to a three-fold pressure rise over the shock wave. In the simulations presented here only the density and volume fraction field are initialised from the previous single-shock simulations, as preliminary simulations highlighted that the the existing velocity field within the mixing layer had only a small influence on the development of the reshocked layer. The velocity impulse imparted by the shock is $\Delta u=29.2$, and the post-shock Atwood number is $\mathrm{At}^{+}=\left(\rho_{2}-\rho_{1}\right) /\left(\rho_{1}+\rho_{2}\right)=0.49$ where $\rho_{2}$ is the heavy gas density and $\rho_{1}$ is the light gas.

A grid refinement study is conducted for the reshock simulation using grid resolutions from $128^{3}$ through to $512^{3}$, where the initial conditions for the coarser grid resolutions were gained by spatially averaging the data from the fine resolution simulation of the development of turbulent mixing layer from the velocity perturbation. Any differences observed between the different grid levels are thus caused by poorer resolution of small scale features in the lower grid resolutions. This reaveraging process was employed as it was reasoned that as amplification of the scales occurs during reshock and that the small scales will have become non-linear and saturated, the effective grid resolution can be decreased.

Periodic boundary conditions are used in the homogeneous direction ( $y$ and $z$ ), whereas a special one-dimensional domain is employed in the direction of shock propagation $(x$ direction). This permits a time-dependent boundary condition in the $x$ direction minimising spurious reflected waves generated at the boundary by simulating a long one-dimensional domain at both ends of the three dimensional domain [50]. All results presented are dimensionless by the pre-reshock integral width $W_{0}$ at the time of reshock which is 0.181 for the narrowband simulation and 0.033 for the broadband simulation, the velocity impulse $\Delta u=29.1575$ imparted by the reshock, and a density equal to one. 


\section{THEORETICAL DISCUSSION}

\section{A. Broadband Perturbations}

The application of current RM theory to reshock of a multimode layer is not straightforward. The layer prior to reshock consists of waves of linear and non-linear amplitudes. Starting at the longest wavelengths, these will not have begun to change in amplitude between the first and second shock, as the growth rate $\dot{a}=\mu \mathrm{At}^{+} k a_{0} \Delta u$ is initially low; $\mu$ is a constant which is typically assumed to be 1 for weak shocks but can reduce at high shock Mach numbers to as little as $0.25[51] ; a_{0}$ is the amplitude of the initial modes. The above means that the reshock will experience the same perturbation shape and amplitude giving the same growth rate exponent at these wavelengths as the first shock. At the shortest length scales the waves will have already saturated, become non-linear, and formed turbulent eddies. It is then expected that these eddies have a kinetic energy spectrum of the form $k^{-5 / 3}$ or for the narrowband case $k^{-3 / 2}[8,40,52]$.

For the longer wavelengths present in the broadband case an estimation of the expected growth rate can be formed, assuming that their amplitude prior to reshock remain linear. This extends the methodology to proposed by Youngs $[1,8,53]$ for the analysis of single shock interaction with a multimode perturbation, following a similar line to that used by Haan [49] (for the Rayleigh-Taylor instability), Brouillette and Sturtevant [54] and Charakhch'yan [55]. The growth of the mixing layer from the initial shock is described based on the power spectrum of the initial perturbation of the form $P(k) \propto C_{1} k^{m}$.

Treating each mode independently, the in-band amplitude of a given wavenumber $|k|$ is initially $a_{k}(t=0) \propto \sqrt{k P(k)}=C_{1}^{1 / 2} k^{(m+1) / 2}$. The velocity corresponding to each wavenumber after the first shock is then $\dot{a}_{k}=C_{1}^{1 / 2} \mu_{1} \mathrm{At}_{1}^{+} \Delta u_{1} k^{(m+3) / 2}$ where the subscript $(.)_{1}$ refers to properties due to the first shock and $\mu \approx 1$ for moderate Mach numbers. Hence the amplitude of each mode for a shock propagating from heavy to light (causing inversion) is given by:

$$
a_{k}(t)=C_{1}^{1 / 2} C_{2} k^{(m+1) / 2}\left(1-\mu_{1} \mathrm{At}_{1}^{+} \Delta u_{1} k t\right), \quad \text {..for } t<t_{R S}
$$

where the mean compression rate $C_{2}=\left(\rho_{1}+\rho_{2}\right)^{-} /\left(\rho_{1}+\rho_{2}\right)^{+}$, and $t_{R S}$ is the time of reshock measured from the time of arrival of the first shock. Fixing the reshock at time $t=t_{R S}$, 
the above equation gives the amplitude of each mode at reshock, under the assumption of linearity. At reshock, this will be in the initial amplitude for the second RM interaction occurring at Atwood $\mathrm{At}_{2}^{+}$and imparting a velocity impulse $\Delta u_{2}$, giving

$$
\begin{aligned}
a_{k}(t) & =a_{k}\left(t_{R S}\right)\left(1-\mu_{2} \mathrm{At}_{2}^{+} \Delta u_{2} k\left(t-t_{R S}\right)\right) \\
& =C_{1}^{1 / 2} C_{2} C_{2, R S} k^{(m+1) / 2}\left(1-\mu_{1} \mathrm{At}_{1}^{+} \Delta u_{1} k t_{R S}\right)\left(1-\mu_{2} \mathrm{At}_{2}^{+} \Delta u_{2} k\left(t-t_{R S}\right)\right)
\end{aligned}
$$

where this is valid only for $t>t_{R S}$. Assuming that a structure of wavelength $\lambda=2 \pi / k$ becomes non-linear in time $t=C_{\lambda} \lambda / \dot{a}_{k}[1,53]$ and that the width of the layer is dominated by the 'just saturating' linear mode, the evolution of the mixing layer width with time is given by making the substitution $W \approx a_{k, R S}=C_{\lambda} \lambda=2 C_{\lambda} \pi / k$ in Eqn. 10

$$
\begin{aligned}
W^{(m+3) / 2}(t)= & C_{1}^{1 / 2} C_{2} C_{2, R S}\left(2 \pi C_{\lambda}\right)^{(m+1) / 2} \times \\
& \left(1-\frac{2 \pi C_{\lambda} \mu_{1} \mathrm{At}_{1}^{+} \Delta u_{1}}{W} t_{R S}\right)\left(1-\frac{2 \pi C_{\lambda} \mu_{2} \mathrm{At}_{2}^{+} \Delta u_{2}}{W}\left(t-t_{R S}\right)\right)
\end{aligned}
$$

Setting $A_{i}=2 \pi C_{\lambda} \mu_{i} \mathrm{At}_{i}^{+} \Delta u_{i}$ gives

$$
W^{(m+7) / 2}(t)=C_{1}^{1 / 2} C_{2} C_{2, R S}\left(2 \pi C_{\lambda}\right)^{(m+1) / 2}\left(W-A_{1} t_{R S}\right)\left(W-A_{2}\left(t-t_{R S}\right)\right)
$$

This can be easily extended to describe several reshocks, and is amenable to numerical solution. If $t$ is sufficiently large then using Eqn. (12) it can be shown that $W(t) \propto t^{2 /(m+7)}$. For a broadband initial condition where $P(k) \propto k^{-2}$, Thornber et al. [8] showed that the first shock would give mixing layer width $W(t) \propto t^{2 / 3}$, and Eqn. (12) shows that following reshock $W(t) \propto t^{2 / 5}$. The expected value of $\theta$ has reduced, and subsequent shocks would clearly reduce this further, until the growth rate predicted by this approach falls below that expected due to the growth of a narrowband layer $(\theta \approx 0.26)$. There is an additional level of dependence on the initial conditions through the coefficient $C_{1}$ (where $P(k)=C_{1} k^{m}$ ); following the first shock $W(t) \propto C_{1}^{1 /(m+5)}$, however after the second shock the sensitivity to $C_{1}$ is reduced as the dependence of the mixing layer width is $W(t) \propto C_{1}^{1 /(m+7)}$.

At intermediate and late times where the initial perturbation range is still linear this approach takes into account both the shape of the initial perturbation, and the evolution of the perturbation prior to reshock (which changes the effective power spectrum of the perturbation). 
Critically, this solution is not applicable to the high wavenumbers which will clearly be non-linear before reshock, hence the predicted growth immediately after reshock will not be accurate. As an example, after each inversion caused by a heavy-to-light reshock the predicted mixing layer width using Eqn. (12) will go to zero (demixing). In reality, this will not occur as the nonlinear modes will not decrease in amplitude. This approximation can be corrected by considering the actual envelope of the mode amplitudes in spectral space, where the above equation is only applied to the modes which are still linear at the time of reshock (an approach previously employed for the Rayleigh-Taylor instability by [49]). Furthermore, it is only valid for initial perturbations where the short wavelengths become non-linear before the long wavelength, i.e. where the perturbation spectrum power exponent $m>-3$. This is not a restrictive requirement given that typical experimentally measured values of $m$ are 0 and -2 .

At very late times Eqn. (12) predicts a reversion to the single shock solution of $\theta \propto$ $2 /(m+5)$ when $W>A_{1} t_{R S}$. This occurs when the modes present in the solution have not yet all saturated and the individual in-band amplitudes for those modes did not change prior to reshock. This is not relevant for the simulations considered here as this late time behaviour would only be present if they were run for approximately seventy times longer, requiring a computationally unfeasible extensive range of initial perturbations.

For the case simulated here, an initial velocity perturbation has been employed, hence the mixing layer is initially flat, meaning that Eqn. (8) becomes $a_{k}(t)=$ $-C_{1}^{1 / 2} C_{2} k^{(m+1) / 2} \mu_{1} \mathrm{At}_{1}^{+} \Delta u_{1} k t$. Propagating this forward and assuming that $\mu_{1}=\mu_{2}=1$ the evolution of this layer should thus be described by

$$
W^{(m+7) / 2}(t)=-C_{1}^{1 / 2} C_{2, R S}\left(2 \pi C_{\lambda}\right)^{(m+1) / 2} A_{1} t_{R S}\left(W-A_{2}\left(t-t_{R S}\right)\right)
$$

An important point to note is that the width $W$ is usually measured as the absolute value (i.e. width is always positive), hence to use the above equation to compare against numerical results we would take the absolute value of $W$. To test the fidelity of the theoretical model, the results of this equation are shown for the evolution of the broadband perturbation (prior to reshock) in Figure 2. Considering that the integral width, defined as

$$
W=\int_{0}^{L_{x}}\left\langle f_{1}\right\rangle\left\langle f_{2}\right\rangle d x
$$


is one of many possible methods of measuring the width of the mixing layer and that no calibrating coefficients have been used, the agreement with experimental data is excellent.

Table I summarises the late time values of $\theta$ for a range of different perturbation power laws of the mixing layer post-first shock perturbation. The perturbation power spectrum after the first shock is essentially proportional to $k^{2}$ times the initial power spectrum. The data is tabulated in this form as it is possible that certain power laws emerge as part of the transition to turbulence - i.e. Kolmogorov scaling $n=-5 / 3$ [40] and $n=-3 / 2$ as proposed by Zhou [52] and observed in simulations by Thornber et al. [8]. For the cases considered here the narrowband perturbation initially has $P(k)=C$ for a narrow range, which would then give a driving range of scales where $P(k) \propto k^{2}$. The broadband simulation has $P(k) \propto k^{-2}$ hence the single-shocked layer should have a power spectrum $P(k) \propto C_{1}$. The final power laws shown in Table I are for $n=2$ and $n=4$ corresponding to two candidate forms of the infrared part of the turbulent kinetic energy spectrum. A key observation is that as the perturbation power spectrum exponent $n$ increases, the predicted value of $\theta$ decreases. As $n$ increases by 2 for each reshock, this implies that eventually the growth rate due to the broadband perturbation will be lower than that due to the growth of a turbulent slab and hence the growth rate will become insensitive to the initial perturbation spectrum.

At late times when the initial perturbations have all saturated, then the layer will continue to grow at the speed of the fastest mode. The variation of velocity of a single mode as a function of time has been examined in detail by many authors since the original analysis of Richtmyer (see $[56,57]$ for recent summaries of theoretical results). Results published by $[51,58]$ indicate that the full velocity predicted by Richtmyer is not reached until $t \Delta u / \lambda=$ 0.3 , and there is an initial overshoot of up to $20 \%$ greater velocity than that predicted by linear theory. Once non-linear, the bubble velocity decreases linearly in the weakly nonlinear phase, and for $t \Delta u / \lambda>>1$ several models predict that the velocity asymptotically approaches zero decaying proportional to $1 / t$ [59-63].

The mixing layer width will be dominated by this velocity until the developing turbulent mixing zone (typically growing around $W \propto t^{0.26}[8]$ ) catches up to the non-linearly saturating modes. In the highly non-linear regime the velocity of the longest wavelengths decays the slowest (as the characteristic time is $\propto \Delta u / \lambda$ ), hence the growth rate of the mixing layer will be dictated by the longest wavelength in the problem. The asymptotic behaviour is then expected to be $W \propto \ln (t)$ [56]. The expected behaviour for a mixing layer, which has 
initially grown from a realistic perturbation would be an initial growth $W \propto t^{2 / 3}$, followed by reshock, which would give first a complex intermediate stage described by Eqn. (13), and then a transition to $W \propto t^{2 / 5}$ (gained by assuming $W<<A_{2}\left(t-t_{R S}\right.$ ) in Eqn. (13)). Finally, when all modes present in the initial perturbation are saturated, there will be a logarithmic transition regime until the developing homogeneous mixing layer growing at a rate $W \propto t^{0.26}$ catches up with the faster growth of the long wavelengths. This behaviour is summarised in Table II.

\section{B. Narrowband Perturbations}

Due to mode coupling and/or entrainment, it is usually postulated that there are eddy structures on the same scale as the layer width present within the mixing layer once the layer has reached a self-similar state after the first shock. If these are present as individual coherent structures they will advect material from the heavy to the light side, and vice versa. This advection process will set up a locally inhomogeneous flow field on the scale of the eddy under consideration, which will then be amplified by RM instability - even though such a structure is not due to a specific single perturbation of that wavelength existing in the initial condition.

This requires a concept of a 'local' Atwood number for a given eddy scale, which would depend on the spatial mean density gradient (at a given time instant in the homogeneous directions) of the mixing layer $\langle d \rho / d x\rangle$, the radius $r$ of the given eddy and the spatial mean density at one extrema of the vortex $\left\langle\rho_{1}\right\rangle$, as shown schematically in Figure 3. The Atwood number of an eddy of that scale would then be

$$
\mathrm{At}=\sqrt{(1-\Theta)} \frac{\left(\left(\left\langle\rho_{1}\right\rangle+2 r\langle d \rho / d x\rangle\right)-\left\langle\rho_{1}\right\rangle\right)}{\left(\left\langle\rho_{1}\right\rangle+\left(\left\langle\rho_{1}\right\rangle+2 r\langle d \rho / d x\rangle\right)\right)}=\frac{\sqrt{(1-\Theta)} r\langle d \rho / d x\rangle}{\left\langle\rho_{1}\right\rangle+r\langle d \rho / d x\rangle} .
$$

The factor $\sqrt{(1-\Theta)}$ is introduced to take into account the reduction of density variance due to the action of molecular mixing, where the molecular mixing parameter $\Theta$ is defined as

$$
\Theta=\frac{\int\left\langle f_{1} f_{2}\right\rangle d x}{\int\left\langle f_{1}\right\rangle\left\langle f_{2}\right\rangle d x}
$$

This is an effective measure of the amount of mixing, which will reduce the growth rate 
of the reshocked layer. As an example, if mixing is perfect (i.e. $\Theta=1$ then there is no instability as the instantaneous density gradients parallel to the interface are zero. A similar argument has been employed in the analysis of the Rayleigh-Taylor instability by Dimonte et al. [64]. Assuming that the gradients within the mixing layer develop self-similarly with respect to the layer width $W$, i.e. $\tilde{x}=x / W, \tilde{r}=r / W$ and $\langle\tilde{\rho}\rangle=\langle\rho\rangle /\left\langle\rho_{1}\right\rangle$ then

$$
\mathrm{At}=\frac{\sqrt{(1-\Theta)} \tilde{r}\langle d \tilde{\rho} / d \tilde{x}\rangle}{1+\tilde{r}\langle d \tilde{\rho} / d \tilde{x}\rangle} .
$$

Next, the amplification of a single vortex is approximated by linear theory (a very coarse assumption), noting that $k=\pi / r$ and that the initial effective amplitude $a \approx C_{r} r$ gives

$$
\frac{\dot{a}_{r}}{\Delta u_{r}}=\tilde{a}_{r}=\sqrt{(1-\Theta)} \frac{\mu C_{r} \pi \tilde{r}\langle d \tilde{\rho} / d \tilde{x}\rangle}{1+\tilde{r}\langle d \tilde{\rho} / d \tilde{x}\rangle} .
$$

where $0 \leq \tilde{r} \leq 1 / 2$ assuming that no structures are present which are larger than the layer width. This expression is essentially just Richtmyer's formula using a local Atwood number $(\sqrt{(1-\Theta)} \tilde{r}\langle d \tilde{\rho} / d \tilde{x}\rangle) /(1+\langle\tilde{r} d \tilde{\rho} / d \tilde{x}\rangle)$ and linking the perturbation amplitude with half it's wavelength. If $\tilde{r} \rightarrow 1 / 2$ then

$$
\tilde{a}_{r} \rightarrow \sqrt{(1-\Theta)} \mu C_{r} \pi \mathrm{At}
$$

as expected from linear theory. For the small scales $\tilde{r} \rightarrow 0$ and $\tilde{a}_{r} \rightarrow$ $\sqrt{(1-\Theta)} \mu C_{r} \pi \tilde{r}\langle d \tilde{\rho} / d \tilde{x}\rangle$. As the local Atwood number (i.e. Atwood for $\tilde{r}<1 / 2$ ) and velocity impulse $\Delta u_{r}$ is always lower than the global Atwood number/velocity impulse then the amplification of the velocities of the long wavelengths will drive the development of a narrowband saturated mixing layer after reshock. It is important to highlight the factor $\sqrt{(1-\Theta)}$, where for a mixing layer developed from a broadband perturbation $\Theta \approx 0.37$ and for a narrowband perturbation it is $\Theta \approx 0.84$. This means that for two mixing layers of identical width at reshock, where one originated from a broadband perturbation and the other from a narrowband perturbation, the initial growth rate should be nearly double for the broadband case. This sensitivity of the initial growth rate to the concentration fluctuations must be taken into account in turbulence modelling approaches.

The assumption that the density gradients lead to an effective perturbation amplitude proportional to the wavelength of the vortex structure is supported by the experimental results of Leinov et al. [13] which indicated that the reshock growth rate was not dependent 
on perturbation amplitude. As long as the mixing layers generated in the experiments were in the same self-similar state (i.e. mean preshock gradients scaled by the mixing layer width) then they would experience the same growth rate regardless of the actual mixing layer width.

It also gives a linear dependence of growth rate on $\Delta u$ in agreement with observations. Using the experimental data of Leinov et al. and assuming that the growth of the layer is dictated by the largest scales where $\tilde{r}=1$, the model constant $\sqrt{(1-\Theta)} C_{r}=0.38 / \pi \approx 0.12$. This justifies the initial assumption that the reshock interaction can be approximated as linear where $\sqrt{(1-\Theta)} \approx 0.4$ from previous narrowband simulations [8]. The form of the growth rate for $\tilde{r} \rightarrow 1 / 2$ is also consistent with that derived by Mikaelian [2]

$$
\dot{a}=C_{M} \operatorname{At} \Delta u
$$

where $C_{M}=0.28$ giving $\sqrt{(1-\Theta)} C_{r} \approx 0.09$. The predicted velocity derived here, if valid, would only apply to the initial growth. It is also applicable to the early stages of reshock of the broadband layer, where it describes the growth of the high wavenumber modes which are already non-linear following the first shock. In both cases, at later times the linear behaviour would become a power law as dissipation of turbulent kinetic energy occurs [14] or as the long wavelengths begin to dominate as described by Eqn. (12).

The parameter $C_{M}$ is very important for the calibration of turbulence models designed to study mixing layer development from the RM instability. In a typical buoyancy-drag model [17], the growth rate of the mixing layer $(V=d h / d t)$ is modelled using

$$
\frac{d V}{d t}=C_{B} A t^{+} g-C_{D} \frac{V|V|}{h} .
$$

where the first term represents turbulence production with a coefficient $C_{B}$ and the second represents drag with a drag coefficient $C_{D}$. During shock interaction it can be assumed that drag is negligible, and integrating this equation over the time taken for the shock to pass through the layer (i.e. $\int g d t=\Delta u$ ) gives

$$
V=C_{B} A t^{+} \Delta u
$$

Comparing Eqn. (22) with Eqn. (19) shows that $C_{B}=C_{M}=\sqrt{(1-\Theta)} \mu C_{r} \pi$. As $\Theta$ is dependent on the form of the initial perturbations prior to the first shock, then according to the derivation presented here $C_{B}$ must also take into account the form of the initial 
perturbations. However, the coefficient $C_{B}$ is also constrained by requirements to correctly represent the case of constant acceleration (Rayleigh-Taylor instability), which gives an approximate solution of the form

$$
h \approx \alpha A t g t^{2}
$$

where $\alpha=0.5 C_{B} /\left(1+2 C_{D}\right)$ and $C_{B} \approx 1 / 2$ [65]. There is a clear trade off between the two conflicting requirements. This difficulty has led to proposed improvements to the standard models to incorporate additional physics, such as the two scale model proposed by Zhou et al [66], which could potentially address some of the issues highlighted here. Numerical simulations within this paper aim to investigate and clarify the variation of $C_{B}$ and $C_{M}$ with respect to the form of the initial perturbation spectrum.

Where the eddies are so small that diffusion renders density variation negligible the amplification of the mode will be similar to that occurring in shock-turbulence interaction, as previously pointed out by Brouillette and Sturtevant [10]. This process is highly unsteady, and the exact physics of the interaction is dependent upon the turbulent Mach number and structure and the incoming shock Mach number [67]. For isotropic turbulence, Linear Interaction Analysis (LIA) results developed in [68] and presented in [69] show that both the shock-normal and shock-parallel velocities are amplified by a factor of $\approx 1.27$ at the shock Mach number used in this study in the inviscid limit. This is under the assumption of isotropy of the initial turbulent flow field, which is not the case here. This would suggest that in the limit of a very well mixed, uniform layer with no large scale structures (relative to the layer width), the reshock process would commence with a narrowing due to the mean compression rate, followed by growth of the mixing layer at approximately 1.27 times the initial rate, assuming that $d W / D t \propto u_{r m s}$.

To investigate this effect, simulations have been run for both the narrowband and broadband perturbations where the velocity field in the turbulent layer prior to reshock was set to zero. In both cases the effect on the properties of the reshocked layer of setting the velocity field to zero was found to be negligible, as shown in Figure 4, indicating that the $\mathrm{RM}$ instability is the dominant process during reshock. There is a twenty-fold increase in turbulent kinetic energy during the interaction, implying that fluctuating velocities have increase by nearly five times which is far higher than that expected from amplification of turbulent velocities alone. This is due to the state of the mixing layer prior to reshock, 
where the turbulent kinetic energy decays at a rate $t^{-2(\theta-1)}$ yet the effective Atwood number is $A t^{+} \propto \sqrt{(1-\Theta)} \rightarrow$ const.. Hence as long as there is sufficient delay between the two shocks, the interaction of the shock and density fluctuations should dominate over shock compression of turbulence. This has important implications for turbulence modelling of these instabilities as many turbulence models (e.g. the $k-\epsilon$ model) rely entirely on the source term $\left(R_{x x} d u / d x\right)$ to model the RM instability. Where density fluctuations dominate this term alone will not be sufficient.

\section{RESULTS}

\section{A. Flow Visualisations}

Figures 5 and 6 show two and three-dimensional visualisations of the volume fraction where the light fluid is coloured black. Compared to the results of a single shock interaction, the flow phenomenology is very similar for both the narrowband and broadband mixing layers, and would most likely be indistinguishable without correct labelling. The threedimensional renderings show that both layers have a very wide range of scales, and the two isosurfaces chosen sandwich a wide, well mixed region.

\section{B. Mixing Layer Width}

The growth of the mixing layer has been computed using the integral width (Eqn. (14), and is plotted in Figure 7. The widths have been non-dimensionalised by the integral width pre-reshock, which for the broadband layer is significantly smaller than the narrowband layer (hence the significantly longer dimensionless time duration). The values of $\theta$ for both perturbation types are detailed in Table III, where they have been computed using a nonlinear regression fit to a power law of the form $W=A\left(t-t_{0}\right)^{\theta}$.

From Figure 7 the integral widths have converged at the two highest resolutions. This is likely the case for the narrowband perturbation, where the values vary very little between the highest two grid resolutions, giving $\theta \approx 0.28 \pm 0.01$. For the broadband case however the value of $\theta$ is still increasing at the highest grid resolution, even though the integral width prediction is close for the highest two resolutions. The value of $\theta=0.36$ for the 
$512^{3}$ resolution simulation, which is slightly below the expected asymptotic value of $\theta=2 / 5$ predicted in Section III A for the case where there is an infinite range of initial perturbation wavelengths. This is not surprising, as one of the conclusions of the single shock studies was that a very wide range of initial perturbations are required to achieve the expected theoretical growth rates [8]. For reshock this constraint will be even more strict given the enhanced growth rates of the individual modes.

The narrowband case grows with $\theta \approx 0.28$ which is marginally faster than the growth prior to reshock (where $\theta \approx 0.26$ ). Interestingly, this is close to the value expected for the reshock of a flow with an initial perturbation spectrum $k^{2}$ which gives $\theta=2 / 7$. According to turbulent theory $[70,71]$, the long wavelengths present in a turbulent layer could have a kinetic energy spectrum proportional to $k^{2}$.

Figure 8 plots both the broadband and narrowband mixing layer width (post-reshock) on the same axis with the growth rate predicted using the Mikaelian model (Eqn. (20)). It has been assumed that $\dot{a}=3 \dot{W}$, i.e. the actual amplitude of the mixing layer is $3 W$. The narrowband growth rate predicted by the Mikaelian model is overestimated, however a revised value of $C_{M}=0.125$ gives a good agreement for the initial linear stage for $0<$ $\Delta u\left(t-t_{R S}\right) / W_{0}<36$. As the pre-reshock value of $\Theta=0.84$, then the coefficient $C_{r}=$ $C_{M} /(\pi \sqrt{1-\Theta})=0.1$ in Eqn. (19). For the broadband case, good agreement is gained with $C_{M}=0.34$, giving $C_{r}=0.14$ for $0<\Delta u\left(t-t_{R S}\right) / W_{0}<18$. A reasonable estimate for the coefficient $C_{r}$ in Eqn. (12) is thus $C_{r}=0.12 \pm 0.02$.

A comparison with the nonlinear theory in Eqn (13) for the broadband perturbation is shown in Figure 9. Although the theoretical solution in Eqn. (13) matched the integral width excellently for the development of the broadband perturbation, the post-reshock mixing layer growth is overestimated by a factor of approximately two when using $C_{\lambda}=1$.. The source of this overestimation could be in the non-linearity of the initial modes, the lack of a wide spectral range in the initial perturbation, or even just that the nature of the mixing layer has changed. The last point is particularly important, as results presented later in this section show that the mixing levels have increased. This will mean that the integral width will not represent the 'actual' width of the mixing layer in the same manner as before reshock, potentially requiring a further correction to the theoretical approach. However, setting the value of $C_{\lambda}=0.27$ gives a very satisfactory match to the evolution of the reshocked layer. 


\section{Mix Parameters}

Two mixing parameters have been investigated, the molecular mixing fraction $\Theta$ and the mixing parameter $\Xi$ which is defined as

$$
\Xi=\frac{\int\left\langle\min \left(f_{1}, f_{2}\right)\right\rangle d x}{\int \min \left(\left\langle f_{1}\right\rangle,\left\langle f_{2}\right\rangle\right) d x} .
$$

where $\left\langle f_{1,2}\right\rangle$ indicates the $y-z$ plane averaged volume fraction of species 1,2 where species 1 is the heavy gas. These parameters are plotted in Figure 10 and 11 for the narrowband and broadband respectively. It is useful to note that in the narrowband simulation prior to reshock the self similar state gives $\Theta=0.84 \pm 0.02$ and $\Xi=0.84 \pm 0.01$. After reshock the asymptotic values have slightly decreased giving $\Theta=0.78 \pm 0.02$ and $\Xi=0.81 \pm 0.02$, indicating that the reaction rate for both a slow and fast reaction would be marginally decreased after reshock. Note that the increase in the mixing parameters seen after reshock is due to inversion of the instability.

Prior to reshock the broadband mixing layer had very low values of the mixing parameters of $\Theta \approx 0.37$ and $\Xi \approx 0.38$ respectively. After reshock the mix levels increase significantly and both $\Theta$ and $\Xi$ are approximately 0.7 . This increase in mixing efficiency of the layer is consistent with the decreased growth rate - a decrease in the growth rate implies a more homogeneously distributed mixing layer.

The early stages of reshock are highlighted in Figure 12 where the narrowband and broadband cases are plotted on the same graph, and the time axis shifted such that the origin is at the arrival of the reshock. In both cases shock transit initially increases the degree of molecular mixing, then as mixing due to the second shock gets underway the molecular mixing parameter drops to a significantly lower level before increasing slightly to an asymptotic value. There is an increase in both $\Theta$ and $\Xi$ before the reshock compresses the mixing layer. This is caused by numerical dissipation of the single-shocked mixing layer due to the transfer of data from the high resolution grid used in the single shock simulation to the coarser grid employed for the reshock. In the broadband case this has a larger effect as the initial conditions are derived from a $720 \times 2048 \times 2048$ grid averaged back to $512^{3}$ which causes an increase in $\Theta$ from 0.37 to 0.54 before the reshock hits the mixing layer. Assuming that the initial growth rate is proportional to $\sqrt{1-\Theta}$, this implies a maximum underestimation of $15 \%$ in the initial growth rate compared to the expected actual result, 
and would also likely result in a reduced actual reshocked value of $\Theta$ due to the increased growth rate. This is considered to be a maximum error as the averaging process will effect mainly the small scales hence should not alter the effective Atwood number for the dominant scales. This is unavoidable given current available computing power.

\section{Turbulent Kinetic Energy}

Figure 13 and 14 show the variation of fluctuating/turbulent kinetic energy with time, defined as

$$
\tilde{u}=\frac{\sum_{y z} \rho u d V}{\sum_{y z} \rho d V}, \quad T K X=\sum_{x y z} \frac{1}{2} \rho(u-\tilde{u})^{2} d V, \quad T K Y=\sum_{x y z} \frac{1}{2} \rho v^{2} d V,
$$

where $(\tilde{.})$ indicates a spatial average over the homogeneous direction. Note that only the $y$-direction turbulent kinetic energy (TKY) is plotted as the $z$-direction is nearly identical due to the inherent symmetry of the problem. The ratio of turbulent kinetic energy in the $x$ and $z$ direction are also plotted as a function of grid resolution. Firstly, there is excellent convergence of the results on different grid levels, which is to be expected as turbulent kinetic energy is a property of the large scales. There are three stages present in both the narrowband and broadband simulations, firstly the kinetic energy increases rapidly during reshock, followed by a readjustment period where TKX decreases more rapidly than TKY and TKZ (which remain nearly constant). The final stage is the decay of all components of the turbulent kinetic energy as a power law in time, where the level of anisotropy (measured by TKX/TKY) remains approximately constant in time. For the narrowband case $T K X / T K Y=1.7 \pm 0.1$, and for the broadband case $T K X / T K Y=$ $1.8 \pm 0.2$, which is higher level than the previous results for a single-shocked layer (where $T K X / T K Y \approx 1.5)$.

Turbulent kinetic energy usually decays as a power law $t^{p}$, where from self-similar arguments it is expected that the decay exponent $p$ of turbulent kinetic energy is proportional to $3 \theta-2[8]$. For the narrowband case this gives $p=-1.16$, and the broadband $p=-0.92$. To investigate this relationship the quantity $T K X \times t^{-p}$ is also plotted in Figures 13 and 14 for the highest grid resolution. This quantity is approximately constant late time for both the narrowband case, giving $T K X \times t^{1.16}=23 \pm 0.5$ for $t \Delta u / W_{0}>40$, and the broadband 
case where $T K X * t^{0.92}=14 \pm 0.5$ for $\left.t \Delta u / W_{0}>400\right)$. This is a strong indication that a self-similar regime has been achieved for the two mixing layers.

\section{E. Turbulent Kinetic Energy Spectra}

Figure 15 shows the variable density turbulent kinetic energy spectra for the narrowband and broadband perturbation prior to reshock, just after reshock, and at late time, defined as

$$
E(k)=\frac{1}{2} \hat{\nu}_{i}^{*} \hat{\nu}_{i}
$$

where $\nu_{i}=\rho^{1 / 2} u_{i}, \hat{(.)}$ indicates the Fourier transform of a quantity, and $\hat{(.)}^{*}$ is the complex conjugate of the transform. The instantaneous spectra are evaluated from two dimensional slices in the homogeneous directions, and Figure 15 shows the spectra in the centre of the mixing layer as a function of $k=\sqrt{k_{y}^{2}+k_{z}^{2}}$.

As observed in Thornber et al. [8] the spectra of the narrowband layer prior to reshock takes a $\mathrm{k}^{-3 / 2}$ form [52]. It is clear from Figure 15 (left) that there is a very strong amplification of over one order of magnitude in kinetic energy for all modes. Immediately following reshock (where the shock has moved fully clear of the layer) there is a $\mathrm{k}^{-5 / 3}$ range present in the spectrum, however at the latest time where self-similarity is achieved the spectrum has returned to a $\mathrm{k}^{-3 / 2}$ form for $10<k<100$, but with more energy at lower wavenumbers than in the pre-reshock case. As the growth rate of the mixing layer is essentially the integral of the kinetic energy spectrum then the slight increase of the growth exponent $\theta$ is most likely due to this increase in low wavenumber energy.

The broadband spectra shown in Figure 15 (right) demonstrate again a clear increase in kinetic energy at all wavelengths, however this is less pronounced than in the narrowband case. At late times the spectrum has a wide range of $k^{-3 / 2}$ between $10<k<70$, and above this the slope is closer to $k^{-5 / 3}$ between $70<k<200$ however it is important to note that these modes are likely to be influenced by the effects of filtering by the grid and numerical viscosity. The late time reshocked layer exhibits a relatively narrow peak, and the peak of kinetic energy (initially shifted to higher wavenumbers) has moved back to lower wavenumbers during the decay process.

The reduced growth rate $\theta$ of the reshocked layer is caused as it no longer exhibits the 
approximately constant spectrum at low wavenumbers, which is present in the pre-reshocked broadband layer and drives the mixing process [8]. There is also a decrease in kinetic energy at the low wavenumbers over reshock which is due to the inversion then subsequent reinversion of these still linear modes.

\section{CONCLUSIONS}

This paper has investigated the flow physics of a reshock Richtmyer-Meshkov induced turbulent mixing layer. It has also proposed a new theoretical description of the reshocked layer by extended the theory of Youngs [1] by assuming that the envelope of the broadband layer growth is described by linearly saturating modes after reshock. There is excellent agreement between the predicted growth rates for the reshocked layer and the results of high resolution Large-Eddy-Simulations, including the predicted decay rate of turbulent kinetic energy based on self-similar assumptions.

The theory of Mikaelian [2] is found to overestimate the initial growth rate of the reshocked narrowband layer by a factor of 2.2 where $C_{M}=0.125$, however it is in reasonable agreement with the broadband case where $C_{M}=0.34$. A modified analytical description for the initial growth rate is proposed which now includes the influence of the density variance through the mixing parameter $\Theta$, with a single coefficient $C_{r}=0.12 \pm 0.02$ giving a good match to both sets of numerical data.

The growth rate exponent of the narrowband layer at late times after reshock $(\theta=0.28)$ is marginally higher than before reshock $(\theta=0.26)$, however there is no significant variation on the flow physics (except for accelerated growth). The broadband layer grows initially with $\theta=0.62$, then transitions to $\theta=0.36$ after reshock (theoretical prediction $\theta=0.4$ ), although the value of $\theta$ is still increasing at the highest grid resolution.

Additionally, it is shown that the post-reshock broadband mixing layer is much more evenly mixed than the pre-reshock layer, where the mix parameter $\Theta$ increases from 0.37 to 0.7 , close to that measured for the narrowband reshocked layer where the post-reshock $\Theta=0.78$. This is due to the decreased growth rate exponent, which permits the layer to develop in a more homogeneous fashion.

This paper has highlighted several potential pitfalls in the modelling of RM instabilities, namely that in some circumstances the increase of turbulent kinetic energy due to shock 
compression of turbulence will be almost negligible compared to that due to the density variation, and most importantly that the form of the initial conditions still influence the nature of the mixing layer following reshock. This has important ramifications in the determination of model coefficients as has been highlighted through the simple buoyancy-drag model, where a dependence on the form of the initial perturbations should be included.However, as discussed in Section III, the effects of initial conditions are expected to diminish after just a few reshocks due to the changing form of the perturbation power spectrum after each shock.

\section{ACKNOWLEDGEMENTS}

The authors would like to acknowledge the financial support from EPSRC, MoD and AWE through the EPSRC(EP/C515153)-JGS(No. 971) project, as well as EPSRC in relation to computational resources (project EP/D053994/1). The computations were run on Cranfield's Astral supercomputer.

[1] D.L. Youngs. Effect of initial conditions on self-similar turbulent mixing. In Proceedings of the 9th International Workshop on the Physics of Compressible Turbulent Mixing, 2004.

[2] K.O. Mikaelian. Turbulent mixing generated by Rayleigh-Taylor and Richtmyer-Meshkov instabilities. Physica D, 36:343-357, 1989.

[3] R.D. Richtmyer. Taylor instability in shock acceleration of compressible fluids. Comm. Pure Appl. Math., 13:297-319, 1960.

[4] E.E. Meshkov. Instability of the interface of two gases accelerated by a shock wave. Fluid Dyn., 43(5):101-104, 1969.

[5] W.D. Arnett, J.N. Bahcall, R.P. Kirshner, and S.E. Woosley. Supernova 1987A. Annu. Rev. Astron. Astrophys., 27:629-700, 1989.

[6] A.S. Almgren, J.B. Bell, C.A. Rendleman, and M. Zingale. Low Mach number modeling of type Ia supernovae. I Hydrodynamics. Astrophys. J., 637:922-936, 2006.

[7] P. Amendt, J.D. Colvin, R.E. Tipton, D.E. Hinkel, M.J. Edwards, O.L. Landen, J.D. Ramshaw, L.J. Suter, W.S. Varnum, and R.G. Watt. Indirect-drive noncryogenic doubleshell ignition targets for the National Ignition Facility: Design and analysis. Phys. Plasmas, 
9(5):2221-2233, 2002.

[8] B. Thornber, D. Youngs, D. Drikakis, and R.J.R. Williams. The influence of initial conditions on turbulent mixing due to Richtmyer-Meshkov instability. J. Fluid Mech., 2010.

[9] V.A. Andronov, S.M. Bakhrakh, E.E. Meshkov, V.N. Mokhov, V.V. Nikiforov, A.V. Pevnitskii, and A.I. Tolshmyakov. Turbulent mixing at contact surface accelerated by shock waves. Sov. Phys. JETP, 44(2):424-427, 1976.

[10] M. Brouillette and B. Sturtevant. Experiments on the Richtmyer-Meshkov instability: Smallscale perturbations on a plane interface. Phys. Fluids, 5(4):916-930, 1993.

[11] B. Vetter and B. Sturtevant. Experiments on the Richtmyer-Meshkov instability on a air $/ \mathrm{sf}_{6}$ interface. Shock Waves, 4(5):247-252, 1995.

[12] B.J. Balakumar, G.C. Orlicz, C.D. Tomkins, and K.P. Prestridge. Simultaneous particleimage velocimetry-planar laser-induced fluorescence measurements of richtmyer-meshkov instability growth in a gas curtain with and without reshock. Phys. Fluids, 20:124103, 2008. doi: $10.1063 / 1.3041705$.

[13] E. Leinov, G. Malamud, Y. Elbaz, L.A. Levin, G. Ben-Dor, D. Shvarts, and O. Sadot. Experimental and numerical investigation of the Richtmyer-Meshkov instability under re-shock conditions. J. Fluid Mech., 626:449-475, 2009.

[14] G.I. Barenblatt, G. Looss, and D.D. Joseph. Nonlinear Dynamics and Turbulence. Pitman Publishing, 1983.

[15] D.L. Youngs. Numerical simulation of mixing by Rayleigh-Taylor and Richtmyer-Meshkov instabilities. Laser Part. Beams, 12:725-750, 1994.

[16] U. Alon, J. Hecht, D. Mukamel, and D. Shvarts. Scale invariant mixing rates of hydrodynamically unstable interfaces. Phys. Rev. Lett., 72:2867-2870, 1994.

[17] D. Layzer. On the instability of superposed fluids in a gravitational field. Astrophys. J., 122(1):1-12, 1955.

[18] A. Rikanati, U. Alon, and D. Shvarts. Vortex model for the nonlinear evolution of the multimode Richtmyer-Meshkov instability at low Atwood numbers. Phys. Rev. E, 58:7410-7418, 1998.

[19] L. Houas and I. Chemouni. Experimental investigation of Richtmyer-Meshkov instability in shock tube. Phys. Fluids, 8(2):614-627, 1996.

[20] J.K. Prasad, A. Rasheed, S. Kumar, and B. Sturtevant. The late-time development of the 
Richtmyer-Meshkov instability. Phys. Fluids, 12(8):2108-2115, 2000.

[21] D.J. Hill, C. Pantano, and D.I. Pullin. Large-eddy simulation and multi-scale modelling of a Richtmyer-Meshkov instability with reshock. J. Fluid. Mech, 557:29-61, 2006. doi:10.1017/S0022112006009475.

[22] M. Latini, O. Schilling, and W.S. Don. Richtmyer-meshkov instability-induced mixing: initial conditions modeling, three-dimensional simulations and comparisons to experiment. In Proceedings of the 10th International Workshop on the Physics of Compressible Turbulent Mixing, July 2006.

[23] O. Schilling, M. Latini, and W.S. Don. Physics of reshock and mixing in single model Richtmyer-Meshkov instability. Phys. Rev. E, 76:026319, 2007. doi:10.1103/PhysRevE.76.026319.

[24] C.W. Barnes, S. H. Batha, A. M. Dunne, G. R. Magelssen, S. Rothman, R. D. Day, N. E. Elliott, D. A. Haynes, R. L. Holmes, J. M. Scott, D. L. Tubbs, D. L. Youngs, T. R. Boehly, and P. Jaanimagi. Observation of mix in a compressible plasma in a convergent cylindrical geometry. Phys. Plasmas, 9(11):4431-4438, 2002.

[25] G. Allaire, S. Clerc, and S. Kokh. A five-equation model for the simulation of interfaces between compressible fluids. J. Comput. Phys., 181:577-616, 2002.

[26] B. Thornber. Implicit large eddy simulation for unsteady multi-component compressible turbulent flows. PhD thesis, Cranfield University, 2007.

[27] D. Drikakis and S. Tsangaris. On the solution of the compressible navier-stokes equations using improved flux vector splitting methods. Appl. Math. Model., 17(6):282-297, 1993. doi:10.1016/0307-904X(93)90054-K.

[28] D. Drikakis. Advances in turbulent flow computations using high-resolution methods. Prog. Aerosp. Sci., 39:405-424, 2003. doi:10.1016/S0376-0421(03)00075-7.

[29] B. Thornber, A. Mosedale, D. Drikakis, D. Youngs, and R. Williams. An improved reconstruction method for compressible flows with low Mach number features. J. Comput. Phys., 227:4873-4894, 2008. doi:10.1016/j.jcp.2008.01.036.

[30] K.H. Kim and C. Kim. Accurate, efficient and monotonic numerical methods for multidimensional compressible flows part II: Multi-dimensional limiting process. J. Comput. Phys., 208:570-615, 2005. doi:10.1016/j.jcp.2005.02.022.

[31] R.J. Spiteri and S.J. Ruuth. A class of optimal high-order strong-stability preserv- 
ing time discretization methods. SIAM J. Num. Anal., 40(2):469-491, 2002 . doi = $10.1137 /$ S0036142901389025.

[32] E.F. Toro. Riemann Solvers and Numerical Methods for Fluid Dynamics. Springer-Verlag, Cambridge, 1997.

[33] B. Thornber, D. Drikakis, R. Williams, and D. Youngs. On entropy generation and dissipation of kinetic energy in high-resolution shock-capturing schemes. J. Comput. Phys., 227:48534872, 2008. doi:10.1016/j.jcp.2008.01.035.

[34] E. Garnier, M. Mossi, P. Sagaut, P. Comte, and M. Deville. On the use of shockcapturing schemes for large-eddy simulation. J. Comput. Phys., 153:273-311, 1999. doi:10.1006/jcph.1999.6268.

[35] B. Thornber, A. Mosedale, and D. Drikakis. On the Implicit Large Eddy Simulation of homogeneous decaying turbulence. J. Comput. Phys., 226:1902-1929, 2007. doi:10.1016/j.jcp.2007.06.030.

[36] S. Dellacherie, P. Omnes, and Fl Rieper. The influence of cell geometry on the Godunov scheme applied to the linear wave equation. J. Comput. Phys., 229:5315-5338, 2010. doi:10.1016/j.jcp.2010.03.012.

[37] S. Dellacherie. Analysis of Godunov type schemes applied to the compressible euler system at low mach number. J. Comput. Phys., 229:978-1016, 2010. doi:10.1016/j.jcp.2009.09.044.

[38] F.F. Grinstein, L.G. Margolin, and W.J. Rider, editors. Implicit Large Eddy Simulation: Computing Turbulent Fluid Dynamics. Cambridge University Press, Cambridge, 2007.

[39] D. Drikakis, M. Hahn, A. Mosedale, and B. Thornber. Large eddy simulation using highresolution and high-order methods. Phil. Trans. R. Soc. A, 367(1899):2985-2997, 2009. doi:10.1098/rsta.2008.0312.

[40] A.N. Kolmogorov. The local structure of turbulence in an incompressible fluid at very high Reynolds numbers. Dokl. Akad. Nauk. SSSR, 30:299, 1941.

[41] M. Hahn and D. Drikakis. Implicit large-eddy simulation of swept wing flow using highresolution methods. Int. J. Numer. Meth. Fl., 47:618-629, 2009.

[42] M. Hahn and D. Drikakis. Assessment of large-eddy simulation of internal separated flow. Journal of Fluids Engineering, 131:071201-071215, 2009.

[43] B. Thornber and D. Drikakis. Implicit large eddy simulation of a deep cavity using high resolution methods. AIAA J., 2008. doi:10.2514/1.36856. 
[44] M. Hahn and D. Drikakis. Large eddy simulation of compressible turbulence using highresolution methods. Int. J. Numer. Meth. Fl., 49:971-977, 2005. doi:10.1002/fld.882.

[45] B. Thornber and D. Drikakis. Implicit large eddy simulation of ship airwakes. Aeronaut. J., 2010.

[46] S. Tissera, V. Titarev, and D. Drikakis. Chemically reacting flows around a double-cone, including ablation effects. AIAA-2010-1285, page 48th AIAA Aerospace Sciences Meeting and Exhibit, 2010.

[47] A. Mosedale and D. Drikakis. Assessment of very high-order of accuracy in les models. Journal of Fluids Engineering, 129:1497-1503, 2007.

[48] G. Fraley. Rayleigh-Taylor stability for a normal shock wave-density discontinuity interaction. Phys. Fluids, 29:376-386, 1986.

[49] S.W. Haan. Onset of nonlinear saturation for Rayleigh-Taylor growth in the presence of a full spectrum of modes. Phys. Rev. A, 39(11):5812-5825, 1989.

[50] B. Thornber and D. Drikakis. Large-eddy simulation of shock-wave-induced turbulent mixing. J. Fluids Eng., 129:1504-1513, 2007. doi:10.1115/1.2801367.

[51] R.L. Holmes, G. Dimonte, B. Fryxell, M.L. Gittings, J.W. Grove, M.S. Schneider, D.H. Sharp, A.L. Velikovich, R.P. Weaver, and Q. Zhang. Richtmyer-Meshkov instability growth: experiment, simulation and theory. J. Fluid Mech., 389:55-79, 1999.

[52] Y. Zhou. A scaling analysis of turbulent flows driven by Rayleigh-Taylor and RichtmyerMeshkov instabilities. Phys. Fluids, 13(2):538-543, 2001.

[53] G. Dimonte, C.E. Frerking, and M. Schneider. Richtmyer-Meshkov instability in the turbulent regime. Phys. Rev. Lett., 74:4855-4858, 1995.

[54] M. Brouillette and B. Sturtevant. Growth induced by multiple shock waves normally incident on plane gaseous interfaces. Physica D, 37:248-263, 1989. doi:10.1016/0167-2789(89)90133-4.

[55] A.A. Charakhch'yan. Reshocking at the non-linear stage of richtmyer-meshkov instability. Plasma Phys. Control. Fusion, 43:1169-1179, 2001. doi: 10.1088/0741-3335/43/9/301.

[56] M. Brouillette. The Richtmyer-Meshkov instability. Annu. Rev. Fluid Mech., 34:445-468, 2002. doi:10.1146/annurev.fluid.34.090101.162238.

[57] M. Latini, O. Schilling, and W.S. Don. Effects of WENO flux reconstruction order and spatial resolutoin on reshocked two-dimensional Richtmyer-Meshkov instability. J. Comput. Phys., 221:805-836, 2007. 
[58] M. Herrmann, P. Moin, and S.I. Abarzhi. Nonlinear evolution of the richtmyer-meshkov instability. J. Fluid Mech., 612:311-338, 2008.

[59] J. Hecht, U. Alon, and D. Shvarts. Potential flow models of Rayleigh-Taylor and RichtmyerMeshkov bubble fronts. Phys. Fluids, 12:4019-4029, 1994.

[60] V.N. Goncharov. Analytical model of nonlinear, single-mode, classical Rayleigh Taylor instability at arbitrary Atwood numbers. Phys. Rev. Lett., 88(13):134502, 2002.

[61] K.O. Mikaelian. Analytic approach to nonlinear Rayleigh-Taylor and Richtmyer-Meshkov instabilities. Phys. Rev. Lett., 80:508-511, 1998. doi:10.1103/PhysRevLett.80.508.

[62] S-I Sohn. Simple potential-flow model of Rayleigh-Taylor and Richtmyer-Meshkov instabilities for all density ratios. Phys. Rev. E, 67:026301, 2003.

[63] Q. Zhang. Analytical solution of layzer-type approach to unstable interfacial fluid mixing. Phys. Rev. Lett., 81:3391-3394, 1998. doi:10.1103/PhysRevLett.81.3391.

[64] G. Dimonte, D.L. Youngs, A. Dimits, S. Wunsch, C. Garasi, M.J. Andrews, A.C. Calder, P. MacNeice, P. Ricker, S. Weber, M. Marinak, A. Robinson, P. Ramaprabhu, B. Fryxell, K. Olson, R. Rosner, J. Biello, L. Dursi, F. Timmes, H. Tufo, Y.-N. Young, and M. Zingale. A comparative study of the turbulent Rayleigh-Taylor instability using high resolution three dimensional numerical simulations: The alpha-group collaboration. Phys. Fluids, 16(5):16681693, 2004. doi: 10.1063/1.1688328.

[65] G. Dimonte and M. Schneider. Density ratio dependence of Rayleigh-Taylor mixing for sustained and impulsive acceleration histories. Phys. Fluids, 12:304-321, 2000.

[66] Y. Zhou, G.B. Zimmerman, and E.W. Burke. Formulation of a two-scale transport scheme for the turbulent mix induced by Rayleigh Taylor and Richtmyer-Meshkov instabilities. Phys. Rev. E, 65(5):056303, 2002. doi:10.1103/PhysRevE.65.056303.

[67] J.H. Agui, G. Briassulis, and Y. Andreopoulos. Studies of interactions of a propagating shock wave with decaying grid turbulence: velocity and vorticity fields. J. Fluid Mech., 524:143-195, 2005 .

[68] H.S. Ribner. Shock-turbulence interaction and the generation of noise. Technical report, NACA, Technical Report No. 1233, 1954.

[69] S. Lee, S.K. Lele, and P. Moin. Interaction of isotropic turbulence with shock waves: effect of shock strength. J. Fluid Mech., 340:225-247, 1997.

[70] P.G. Saffman. A model for inhomogeneous turbulent flow. J. Fluid Mech., 27:581-593, 1994. 
[71] A. Llor. Invariants of free turbulent decay. arXiv:physics/0612220v1 [physics.flu-dyn], 2006. 
Figures 
Turb. layer

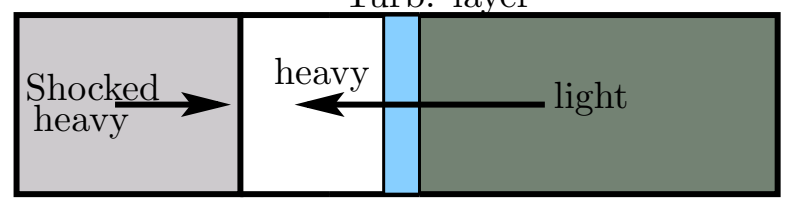

FIG. 1: 


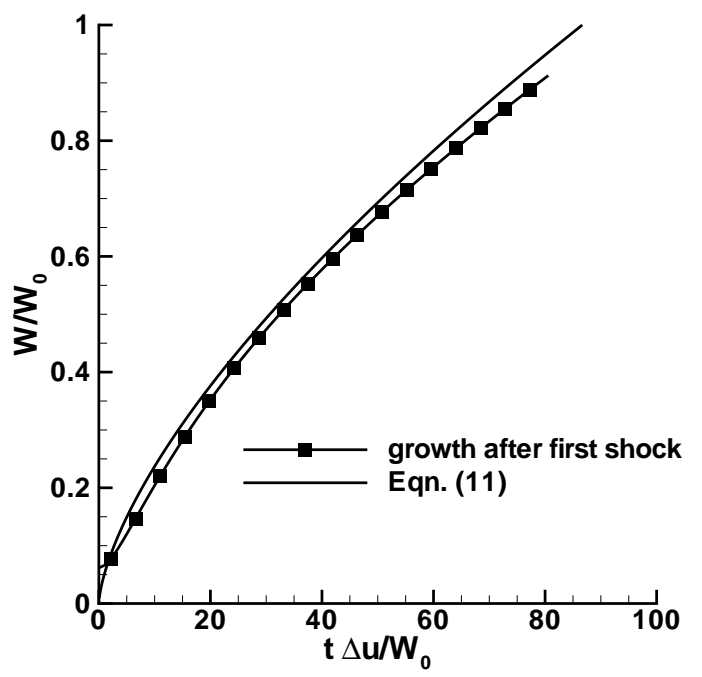

FIG. 2: 

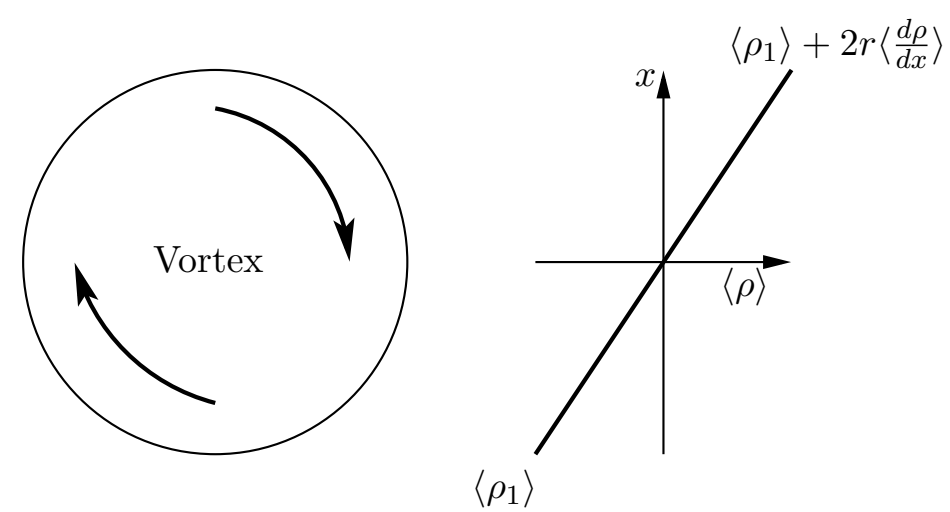

FIG. 3: 


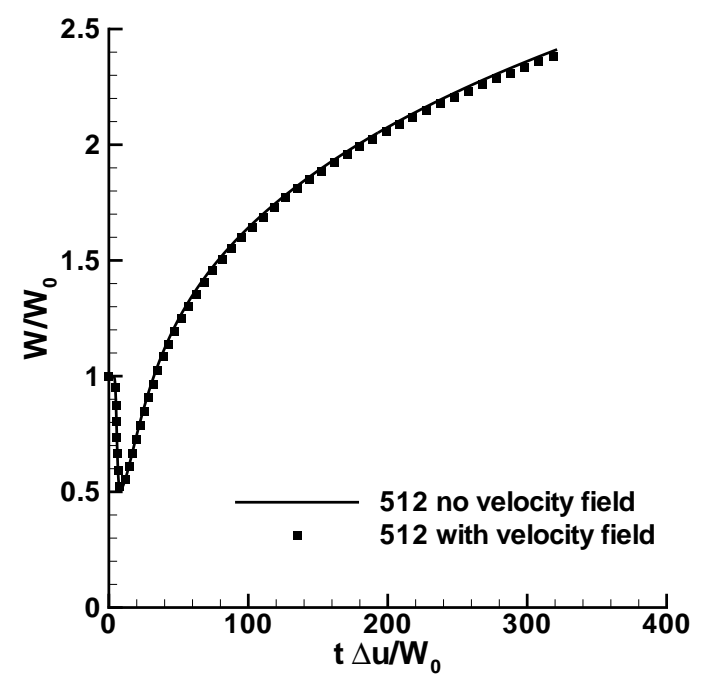

FIG. 4: 

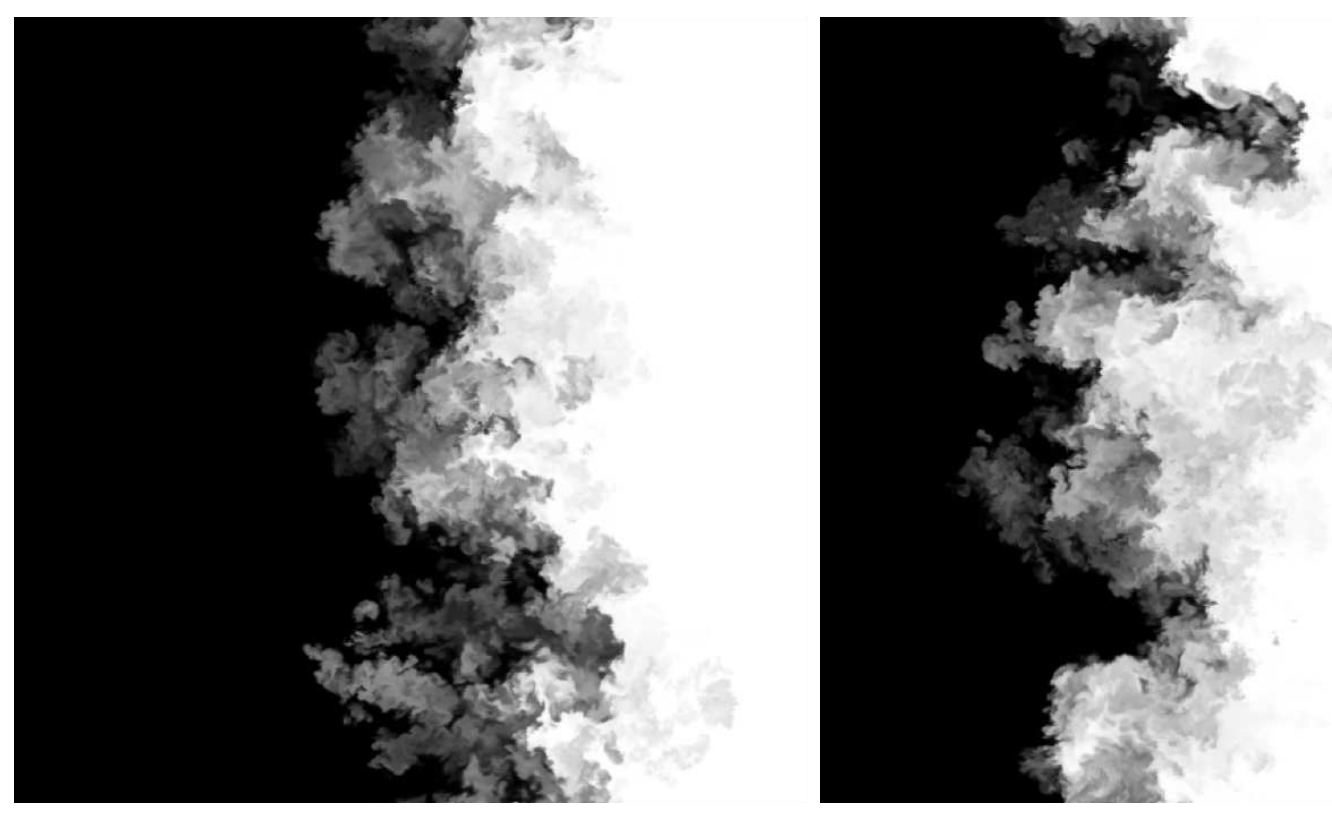

FIG. 5: 

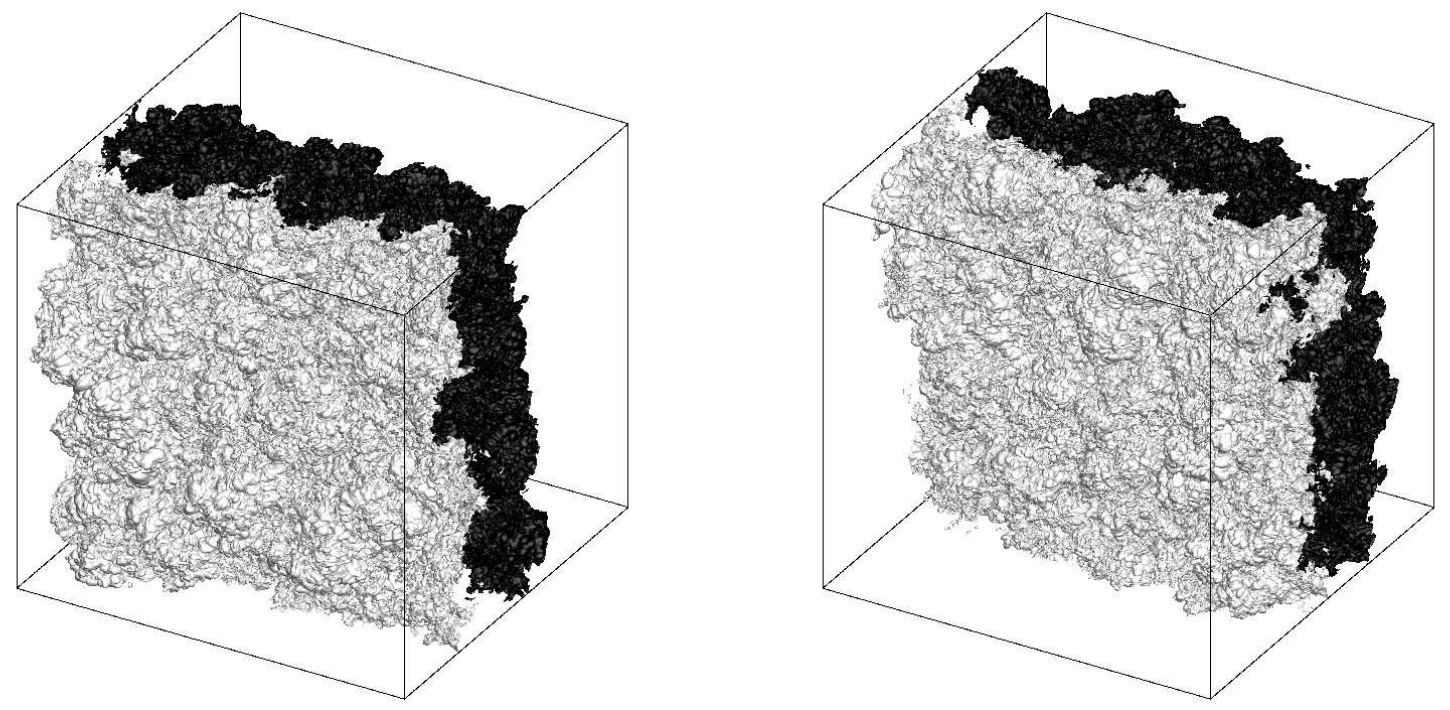

FIG. 6: 

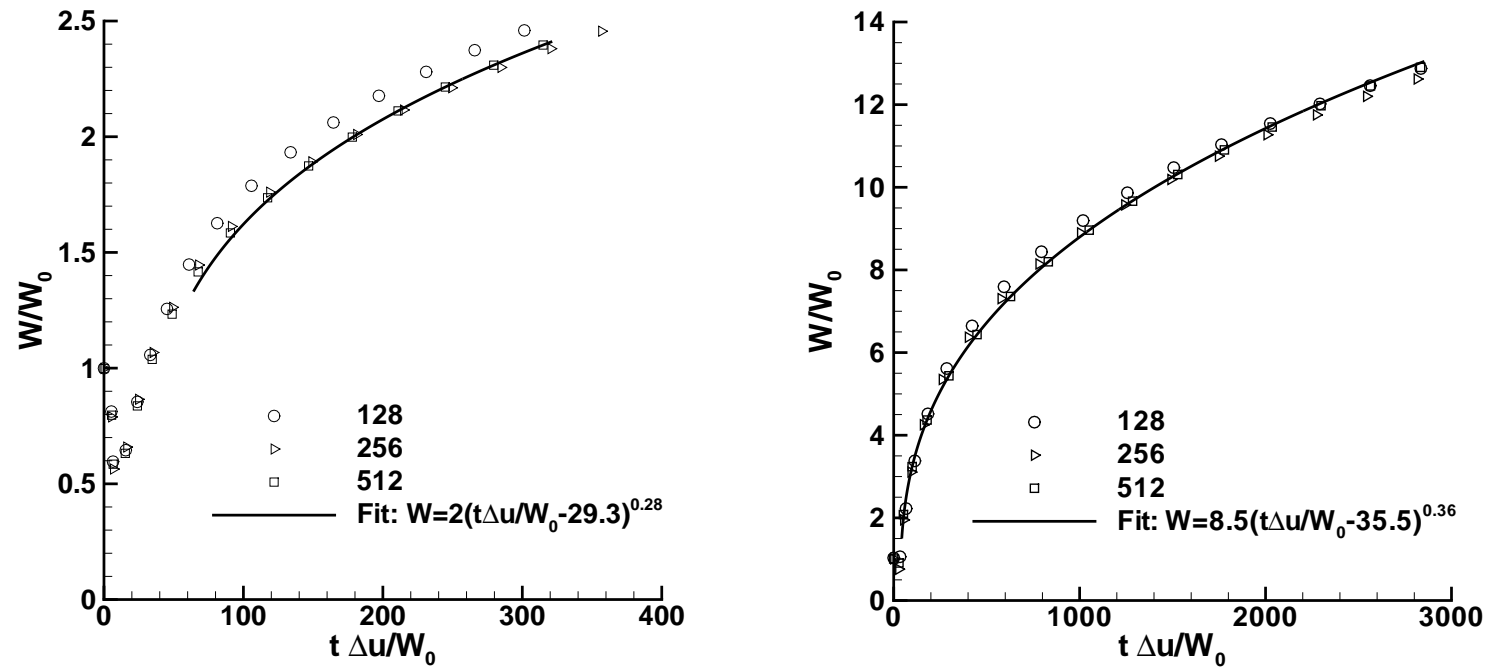

FIG. 7: 


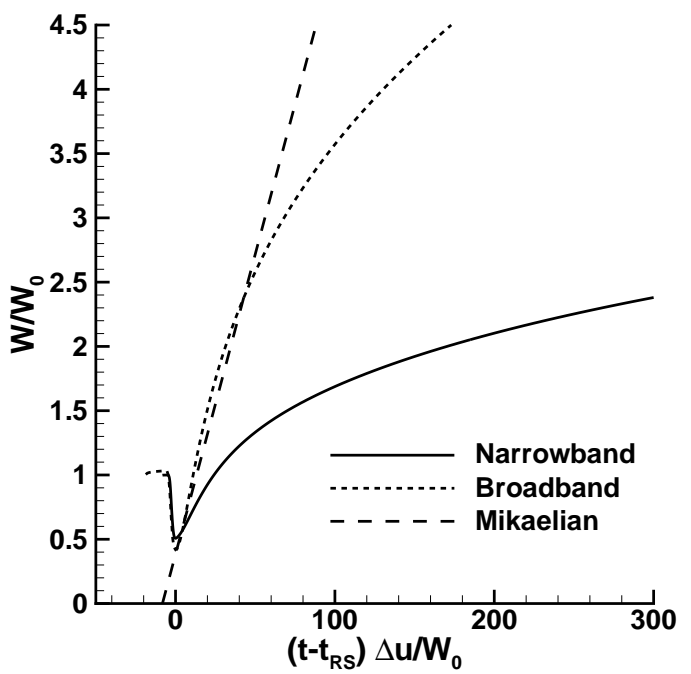

FIG. 8: 


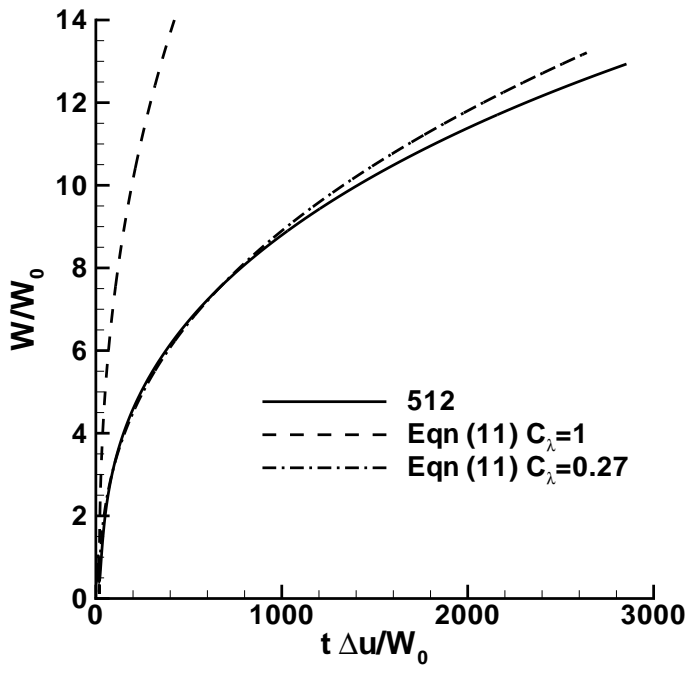

FIG. 9: 

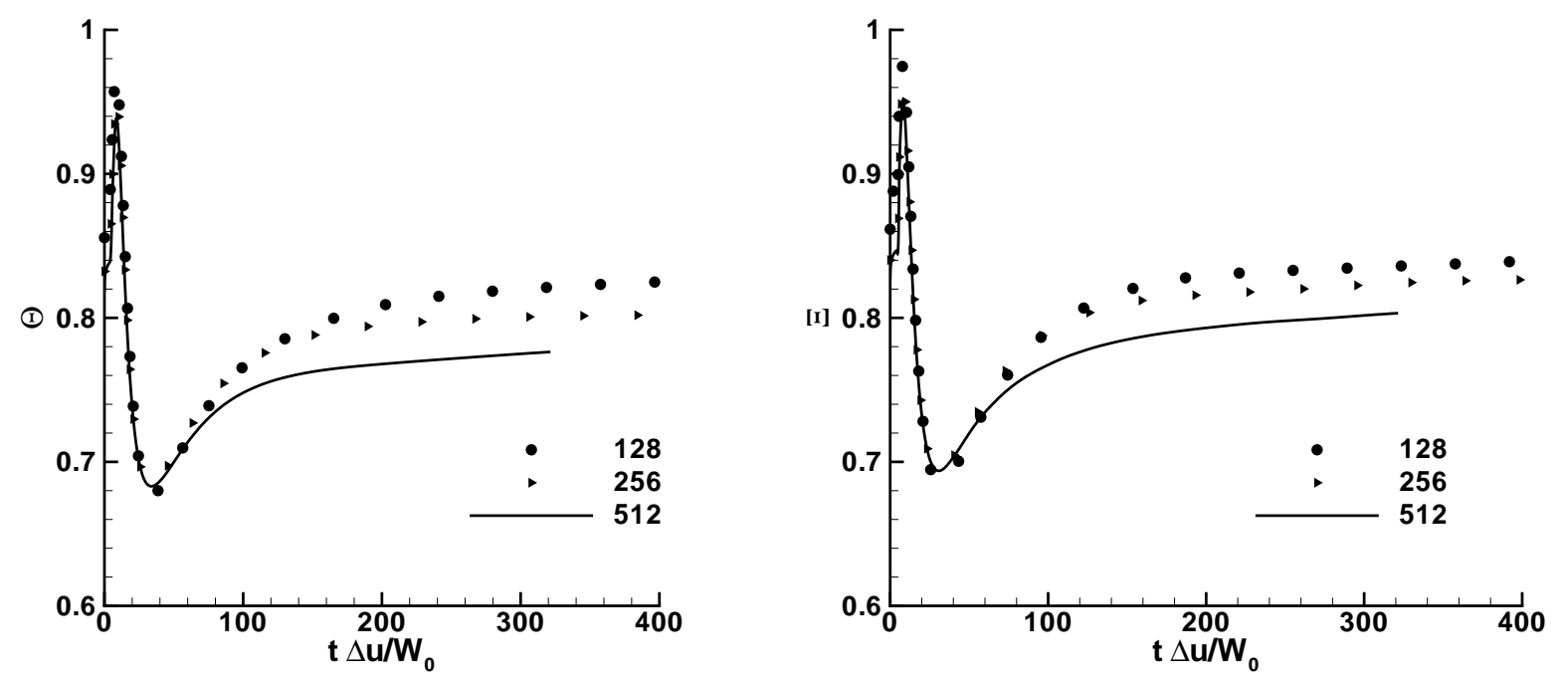

FIG. 10: 

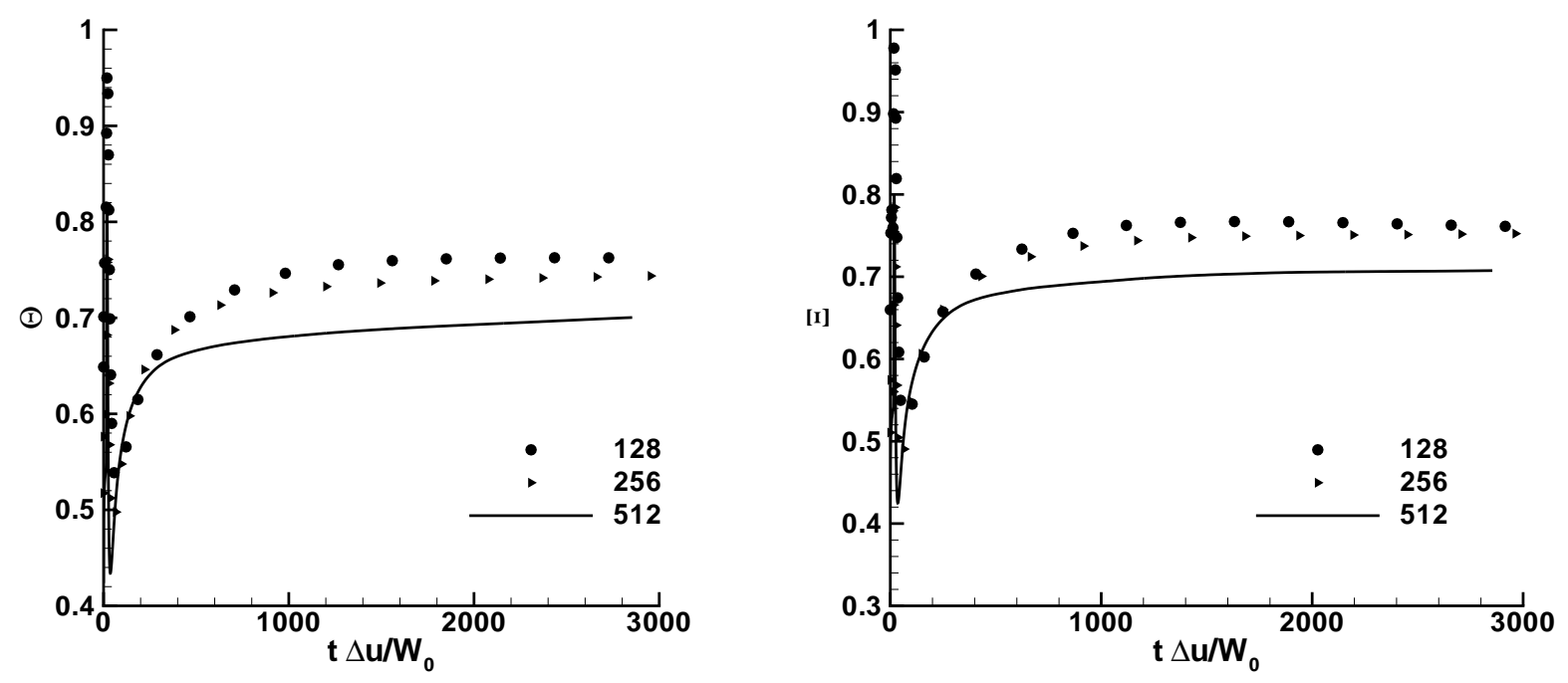

FIG. 11: 

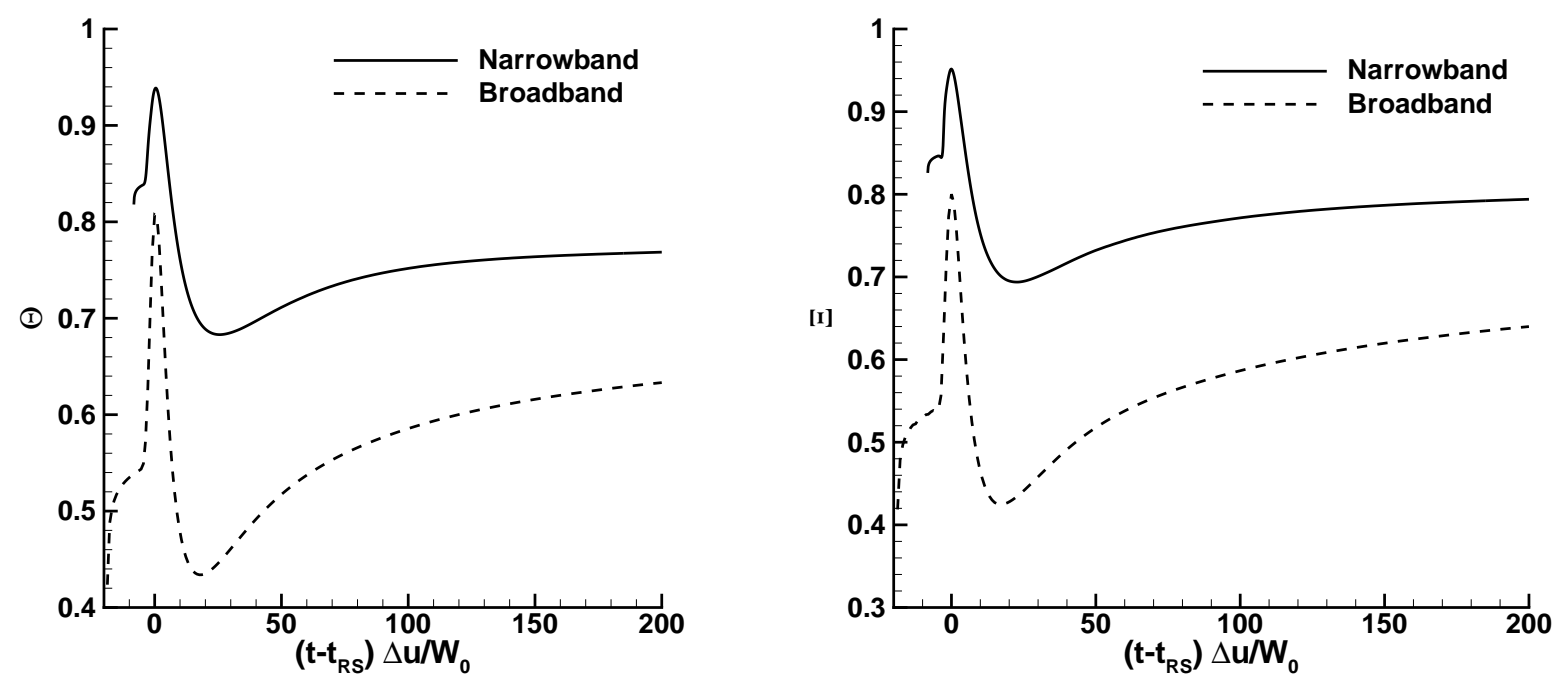

FIG. 12: 

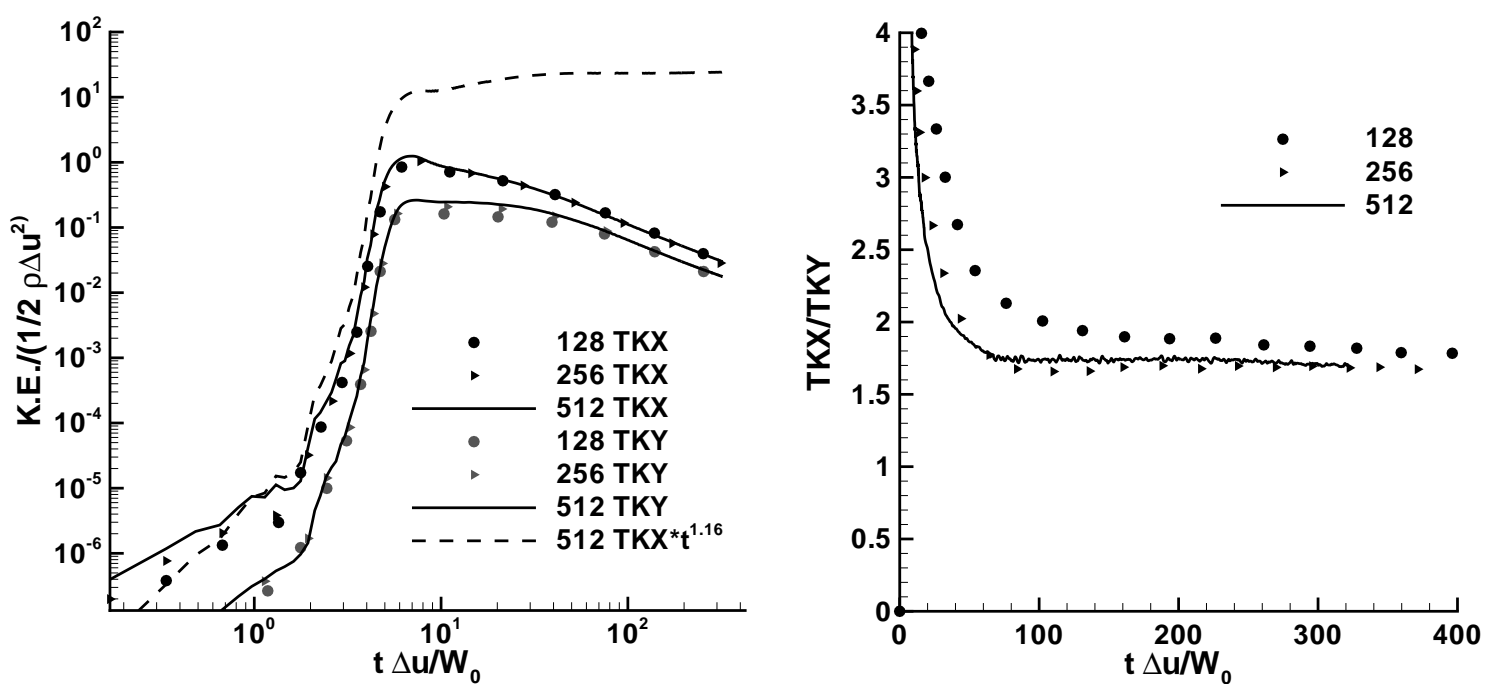

FIG. 13: 

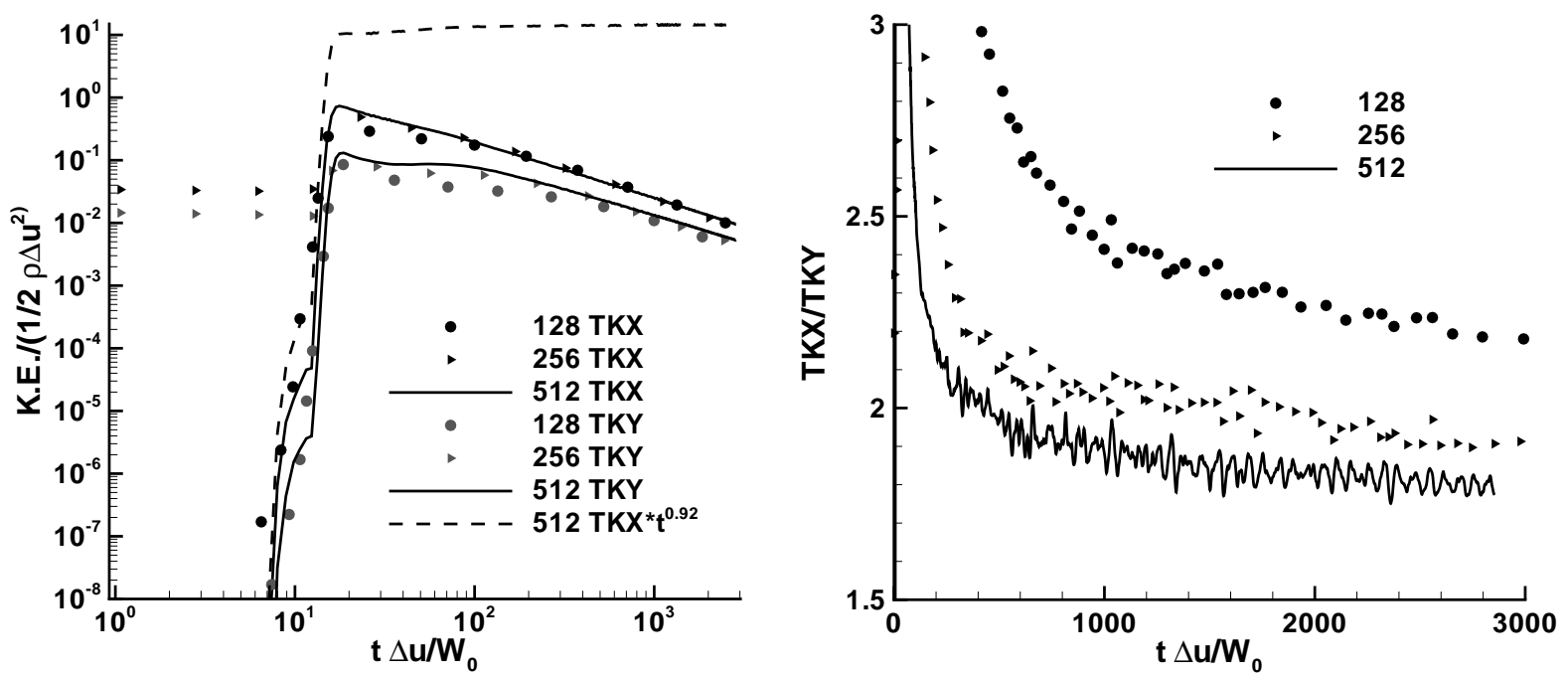

FIG. 14: 

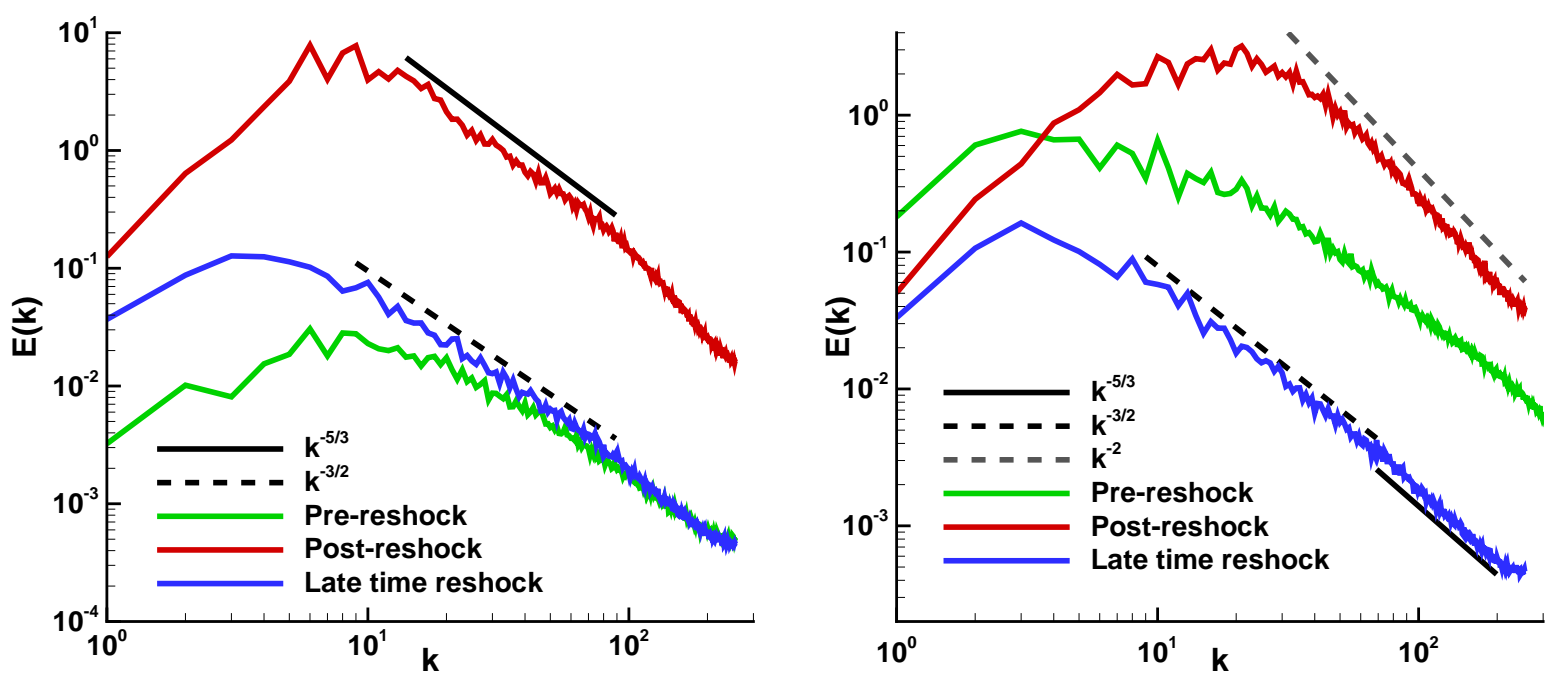

FIG. 15: 


\section{Captions}

Figure 1: The initial condition for the reshock study. Note that the computational domain size is $2 \pi \times 2 \pi \times 2 \pi$. The arrows indicate the initial direction of motion of the fluids.

Figure 2:Comparison of the theoretical prediction for mixing layer width with data from a very high resolution single-shock broadband RM instability preformed by Thornber et al. [8]

Figure 3:Schematic of a typical eddy in a turbulent mixing layer across which there is a spatial mean density gradient $\langle d \rho / d x\rangle$.

Figure 4:Evolution of integral width for the $512^{3}$ reshocked narrowband simulation with and without a velocity field in the pre-reshock mixing layer

Figure 5:Two-dimensional slices through the reshocked narrowband (left) and broadband (right) mixing layers in the self-similar regime. Note that the light fluid is black

Figure 6:Three-dimensional visualisations of isosurfaces of volume fraction (0.01 and 0.99) for the reshocked narrowband (left) and broadband (right) mixing layers in the self-similar regime

Figure 7:Evolution of integral width versus time for the narrowband mixing layer (left) and broadband (right). Included on the narrowband figure is a comparison with the theory of Mikaelian [2]

Figure 8:Evolution of integral width versus time for both mixing layers, where the time scale has been shifted to set $t_{R S}=0$

Figure 9:Evolution of integral width versus time for the broadband mixing layer at the highest resolution compared to the theoretical approaches outlined in Section III

Figure 10:Evolution of mix parameters versus time for the narrowband mixing layer 
Figure 11:Evolution of mix parameters versus time for the broadband mixing layer

Figure 12:Evolution of mix parameters versus time in the early stages at $512^{3}$ grid resolution

Figure 13:Evolution of kinetic energy(left) and the ratio of $x$ to $y$ direction kinetic energy (right) versus time for the narrowband mixing layer

Figure 14:Evolution of kinetic energy(left) and the ratio of $x$ to $y$ direction kinetic energy (right) versus time for the broadband mixing layer

Figure 15:Evolution of the kinetic energy spectra for the narrowband (left) and broadband case (right) during reshock and at late time 


TABLE I:
\begin{tabular}{c|c|c|c|c|c}
$n$ & $-5 / 3$ & $-3 / 2$ & 0 & 2 & 4 \\
\hline$\theta$ & $3 / 5$ & $4 / 7$ & $2 / 5$ & $2 / 7$ & $2 / 9$
\end{tabular}

\section{Tables}

\section{Captions}

Table 1:Tabulated values of the late time value of $\theta$ based upon Eqn. (13) as a function of the post-first shock power spectrum $P(k)=C k^{n}$ of the single-shocked layer. Note that $n=m+2$ for scales generated by RM instability

Table 2: Different growth rate regimes expected for the RM broadband layer before, during and after saturation of all initial perturbations

Table 3: Variation of $\theta$ for the narrowband (N) and broadband (B) case as a function of grid resolution 
TABLE II:

\begin{tabular}{c|c|c|c|c|c} 
Stage & First shock & $\begin{array}{c}\text { Reshock } \\
t \approx t_{R S}\end{array}$ & $\begin{array}{c}\text { Reshock } \\
t \Delta u / \lambda_{\max }<1\end{array}$ & $\begin{array}{c}\text { Late time } \\
t \Delta u / \lambda_{\max }>1\end{array}$ & $\begin{array}{c}\text { Latest time } \\
t \Delta u / \lambda_{\max }>>1\end{array}$ \\
\hline Behaviour & $\theta=2 /(m+5)$ & Complex, Eqn. (13) & $\theta=2 /(m+7)$ & $W \propto \ln t$ & $\theta \approx 0.26$
\end{tabular}


TABLE III:

\begin{tabular}{c|c|c}
$N$ & $\theta(\mathrm{N})$ & $\theta(\mathrm{B})$ \\
\hline $128^{3}$ & 0.26 & 0.296 \\
$256^{3}$ & 0.275 & 0.339 \\
$512^{3}$ & 0.28 & 0.362
\end{tabular} 